\title{
III.
}

Aus der chirurgischen Universitätsklinik in Berlin.

(Direktor: Geheimrat Prof. Dr. Bier.)

\section{Zur Behandlung der Arthritis gonorrhoica der großen Gelenke mittels Stanungshyperämie.}

Von

Dr. W. Baetzner.

Im Jahre 1894 machte Bier (1) $\left.{ }^{1}\right)$ seine ersten Mitteilungen über die Behandlung der Arthritis gonorrhoica mittels Stauungshyperämie. Seine Angaben wurden lange Zeit nicht nachgeprüft, wenigstens sind keine Veröffentlichungen darüber erfolgt. Erst im Jahre 1903 berichtete $\mathrm{Habs}(2)$ über 4 einschlägige Beobachtungen, bei denen er "die besten Erfolge" sah. Seiner Veröffentlichung folgte eine Reihe anderer Arbeiten. So referierte Luxemburg (3) über 8 Fälle, Laqueur (4) über 5 Fälle; sie heben die Schmerzlinderung der Staungshyperämie hervor und eine Abkürzung der Heilungsdauer. Ihrem Urteil schließen sich Tillmans (5), Tieling (6) und Bum an. Letzterer betont besonders, daß Ankylosen nach Einführung der Stauungstherapie bei der Arthritis gonorrhoica zu den Seltenheiten gehören. Über eine größere Beobachtungsreihe verfügt Hirsch (8), der übrigens, wie wir später sehen werden, viel zu kurze Zeit staut. Auch er erreichte gute Erfolge, will aber von der Stauung bezüglich der Gelenkfunktion und der Krankheitsdauer keine Besserung gegenüber der früher geübten Behandlung gesehen haben. In nenester Zeit spricht besonders Leyden (9) der Staung das Wort und bezeichnet sie als entschiedenen Fortschritt in der Therapie der Gelenkerkrankungen. Er beobachtete neben der Abkürzung der Krankheitsdauer eine bessere und schnellere Wiederherstellung der Gelenkfunktion, und wünscht der Bierschen Stauung eine noch ausgiebigere Anwendung. Treupel endlich berichtet über 12 Fälle,

1) Diese Zahlen beziehen sich auf das am Schlusse dieser Arbeit befindliche Literaturverzeichnis. 
Arapow im Russki Wratsch über 7 Fälle und Matthies über 10 Fälle aus der Kieler Klinik; sie alle bestätigen die guten Erfolge Biers.

In unserer Klinik wurden seit 1904 auf Anregung v. Bergmanns 1) die gonorrhoischen Gelenkerkrankungen der Stauungstherapie unterworfen, und diese Behandlungsmethode ersetzte in der Folgezeit die früher geübte Immobilisierung.

Da die Statistik kritisch beobachteter, mit Stauung behandelter gonorrhoischer Arthritiden in der Literatur sehr spärlich ist, so erscheint es berechtigt, die in unserer Klinik gesammelten Erfahrungen unter Zugrundelegung von 40 Krankheitsfällen mit ausfülırlichen Krankheitsgeschichten kurz mitzuteilen.

Bezüglich der Technik der Bindenstauung hielten wir uns an die Vorschriften Biers. Oberhalb des erkrankten Gelenkes, beim. Hand- und Ellenbogengelenk am Oberarm, beim Knie- und Fußgelenk am Oberschenkel, wird eine dünne, durchsichtige, ca. $6 \mathrm{~cm}$ breite Gummibinde in Touren so angelegt, daß die Bindengänge sich nicht vollständig decken, sondern einen größeren Teil des Gliedes umfassen; zweckmäßig legt man einige Mullbindengänge unter die Binde, die dann mit zwei an ihrem Ende angenähten Bändchen festgebunden wird.

Zur Schulterstauung verwendet man Stücke eines weichen, fingerdicken Gummischlauches, der bis auf seine beiden Enden zur Vermeidung von Druck mit Filz umfüttert wird. Der Schlauch wird von der Achselhöhle aus um die Schulter gelegt, und nachdem er so fest angezogen, daß eine starke venöse Stauung im Arm entsteht, durch eine Klemme oder einen Bindenknoten geschlossen.

Der geschlossene Gummischlauch wird nun weit über die erkrankte Schulter gezogen und durch ein Gurtband, das nach vorn und hinten über den Thorax ver]äuft und unter der gesunden Achselhöhle zusammengeschnallt wird, am Abgleiten verhindert.

Durch entsprechendes Anziehen der Binde resp. des Schlauches versucht man einen solchen Grad von Stauung zu erreichen, daß die gestaute Extremität ein heißes feurigrotes Ö̉dem bei gut fühlbarem Pulse zeigt. Schmerzen und Blaufärbung der Glieder müssen unter allen Umständen vermieden werden. In akuten Krankheitsfällen erreicht man dies leicht und schnell, schwieriger und oft erst

1) Dr. Guleke, derzeit. Assistent an der chir. Universitätsklinik in Straßburg, danke ich an dieser Stelle für die Überlassung seines Materials. 
nach wiederholten Versuchen im subakuten oder chronischen Stadium, wo die Entzündungserscheinungen mehr oder weniger abgelaufen sind.

In dieser Weise haben wir bei unseren ersten Versuchen unter dem Einfluß der ersten Mitteilungen Biers kürzere Zeit gestaut, 1 bis 12 Stunden täglich, sind aber später zu Dauerstauungen von 20 bis 22 Stunden übergegangen.

Die „Minutenstauung“, wie sie Hirsch ausübt, halten wir für ganz unzureichend; er hat es der zu kurzen Staungszeit zuzuschreiben, wenn er keine besseren Resultate gegenüber seiner früheren Behandlung sah.

Jedenfalls müssen wir nach unseren Erfahrungen strikte an der Forderung einer langedauernden, am zweckmäßigsten 20 bis 22 stündigen Stauung festhalten. Die Frage, wann man mit der Stauung im einzelnen Falle aufhören soll, erledigt sich im allgemeinen von selbst, indem man allmählich, sobald nämlich der Entzündungsreiz fortfällt, keine heiße Stauung mehr erreicht, was hinwiederum ein Zeichen dafür ist, daß die Entzündungserscheinungen im Abklingen begriffen sind. Es ist jedoch ratsam, die Stauung nicht $\mathrm{zu}$ früh auszusetzen.

Obwohl wir nun diese Dauerstauung oft wochenlang fortgesetzt haben, haben wir nie ernstere Folgeerscheinungen gesehen. Zuweilen erlebt man Blasenbildungen am Rand der Binde, leichte Epitheldefekte unter dem Schulterring, die aber bei guter Hautpflege (Abwaschen mit Spiritus, Pudern), guter Unterfütterung des Schlauches und der Binde zu vermeiden sind. Bei längerer Stauung tritt hier und da eine zirkuläre Atrophie der Muskulatur an der Konstriktionsstelle ein; prophylaktisch ist darauf $\mathrm{zu}$ achten, daß man die Umschnürungsstelle häufiger wechselt, auch weicht die Atrophie stets, wenn auch langsam, sobald die Binde fortfällt.

Wenn auch die Bindenstauung ambulant ein größeres $\mathrm{Maß}$ von Vorsicht erfordert, so gewannen wir doch bei dem vorwiegend poliklinischen Material den Eindruck, daß die Stanungstherapie bei der gonorrhoischen Arthritis sich sehr wohl poliklinisch durchführen läßt. Der Kranke lernt bald, worauf es ankommt, und ist sogar später imstande, den Arzt bei der Dosierung der Binde zu unterstützen. Natürlich bleibt es stets wünschenswert, die schweren Formen ins Krankenhaus aufzunehmen.

Nach dem Abklingen der akuten Entzündungserscheinungen haben wir zur Unterstützung der Stauung und später allein zur 
Nachbehandlung andere physikalische Maßnahmen herangezogen, besonders die Heißluftbehandlung und Massage.

Immer und immer wieder machen wir die Erfahrung, daß Kranke außerhalb ohne Erfolg gestaut wurden, während wir dieselben sofort erfolgreich stauten. Der Grund für die Mißerfolge liegt gewöhnlich an der falschen Technik; entweder wird zu kurz gestaut, oder aber die Binde zu fest angezogen, so daß der Kranke statt Schmerzlinderung eine Schmerzsteigerung erfährt. Ein präzises Innehalten der von Bier gegebenen Vorschriften, der gute Wille, technische Schwierigkeiten zu überwinden, werden manchen Mißerfolg in der Folgezeit ausschalten.

Wenn wir nun unter kritischer Verwertung unserer Krankengeschichten der Frage näher treten, was die Stauungsbehandlung bei der sozial so wichtigen Erkrankung der Arthritis gonorrhoica leistet, so müssen wir das alte therapeutische Regime, wie es in der Immobilisierung durch Schiene oder Gips fast ausschließlich den Heilplan beherrscht, zum Vergleiche heranziehen.

Die Schwere unseres Krankheitsbildes ist bedingt durch die enorme Schmerzhaftigkeit; das Allgemeinbefinden leidet sehr unter derselben, wochenlang entbehrt der Kranke des Schlafes. Hier brachte die Ruhigstellung durch Verbände auch unter Zuhilfenahme von Analgetika keine andauernde Erleichterung.

Die einfache Binde dagegen bringt oft schon nach Verlauf weniger Minuten eine wesentliche Herabminderung der Schmerzen, die, solange die Binde liegt, auch anhaltend ist. Nach kurzer Zeit weicht der Schmerz ganz, um während der Behandlungszeit kaum wieder aufzutreten. Mit der Schmerzlinderung setzt auch der Schlaf wieder ein, die Binde bewährt sich, wie Bier sagt, als bestes Schlafmittel.

Nur in ganz vereinzelten Fällen versagt die Binde bezüglich ihrer schmerzlindernden Wirkung, besonders dann, wenn ein kräftiges Stauungsödem sich nicht erzielen läßt. Wiederholte Versuche, Hin- und Herprobieren führt schließlich doch zum Ziel.

Im Falle 32 und 35 traten als Komplikation schwere Neuritiden auf. Während in dem einen Fall, bei dem der Radialis und UInaris in seinem ganzen Verlauf auf Druck äußerst schmerzhaft war, die Schmerzen schon nach einigen Tagen verschwanden, bestanden dieselben im Fall 32 trotz der Binde mehrere Wochen. Auch Bier macht in seinem Buch darauf aufmerksam, daß eine Neuritis möglicherweise die Ursache für das Mißlingen der Staungsbehandlung abgeben könne.

Deutsche Zeitschrift f. Chirurgie, 93. Bd. 
Der klinische Verlauf der Erkrankung war nun unter der Fixationsbehandlung ein chronischer. Heilte mitunter der Hydrops serosus schon nach einigen Tagen aus, so wären die phlegmonösen Formen um so hartnäckiger; wochen- und monatelang blieben die schweren Entzündungserscheinungen mit ihrer deletären Wirkung auf den Gelenkapparat und die benachbarten Sehnen stationär.

Unter der Staungstherapie ist ganz $z$ weifellos eine raschere Beeinflussung der entzündlichen Symptome zu konstatieren. Rötung, Schwellung, Ödem anfangs absichtlich gesteigert, schwinden auffallend rasch und machen früh normalen Verhältnissen Platz, mitunter in so kurzer Zeit, daß es berechtigt erscheint, nicht bloß an einen mechanischen Effekt der Stauung zu denken, der im Sinne eines Verdünnungsmittels endgiftend wirkt, sondern an eine spezifische Beeinflussung der Krankheitsnoxe. Dieser raschen Beseitigung des entzündlichen Stadiums ist es auch zu danken, wofern man von der Frage nach einer Mischinfektion absehen will, daß wir die allerdings seltenen Vereiterungen der Gelenke nicht erlebt haben, und damit auch Incisionen und Drainage entbehren konnten.

Eine exquisite Tendenz zur Versteifung und Ankylosierung gibt der gonorrhoischen Gelenkentzündung ihr charakteristisches Gepräge. Das anfangs durch Nervenreiz und Muskelkontraktur festgehaltene Gelenk versteift durch Schrumpfung des infiltrierten artikulären und periartikulären Gewebes und ankylosiert durch Verwachsen der ihres Knorpels verlustig gegangenen Gelenkenden.

Diese große Neigung zur Versteifung hat die alte Behandlungsweise mit dem Dogma der Immobilisierung aufs nachhaltigste gefördert. Der behandelnde Arzt war sich unklar über die Wahl des Zeitpunktes, wann er mit der Immobilisierung aufhören, wann er mit passiven Bewegungen beginnen sollte. Nur zu häufig büßte er vorzeitige Bewegungen mit einer Exazerbation des Prozesses, die ihn zwang, das kranke Gelenk aufs neue dem Gipsverband zu überantworten, wobei er einer mit um so größerer Wahrscheinlichkeit einsetzenden Versteifung untätig zusehen mußte. So kam König in der Charité allmählich dazu, den Zeitpunkt der Mobilisierung auf Kosten der Gelenkfunktion weiter hinauszuschieben (Bennecke).

Die Stauungshyperämie hat nun hier einen ganz wesentlichen Wandel geschaffen. Ihre hervorragend schmerzlindernde Wirkung ermöglicht selbst bei den schwersten phlegmonösen Formen ganz frühzeitig mitunter sofort passive und aktive Bewegungen. Bei allen unsern Kranken haben wir ohne Schaden, ohue Schmerzen, 
Zur Beh. d. Arthr. gonorrh. d. großen Gelenke mittels Stauungshyperämie. 51

ohne eine Verschlimmerung oder ein Wiederaufflackern des Prozesses zu erleben, frühzeitig Bewegungsübungen vorgenommen.

Diese frühzeitige Mobilisierung beseitigt schnell die reflektorische Muskelkontraktur, bekämpft die Muskelatrophie und verhindert ein Verwachsen der Gelenkenden; kurz sie ist die conditio sine qua non für die Sanierung der Gelenkfunktion.

Für die Würdigung der Frage aber, welche Methode bei der Behandlung der gonorrhoischen Gelenkmetastasen bessere Resultate gibt, sind besonders zwei Faktoren von ausschlaggebender Bedeutung: die Krankheits- und Behandlungsdauer und die Gelenkfunktion.

Bezüglich beider Punkte ist in der Literatur, was die Erfolge der früher geübten Behandlung anbelangt, kein brauchbares zum Vergleich heranzuziehendes Zahlenmaterial vorhanden. Ein Versuch, aus unserem früheren poliklinischen Material hierüber Aufschluß zu erhalten, mußte aufgegeben werden, da man bezüglich Krankheitsdauer und -schwere auf die Angaben der Patienten angewiesen war; doch ergab eine kleine Stichprobe unter 20 Fällen 5 Ankylosen. Im allgemeinen war die Behandlungsdauer eine äußerst langwierige; umfaßte oft mehrere Monate. Die funktionellen Resultate waren wenig erfreulich; oft genug blieb ein dauernd schmerzhafter Zustand des erkrankten Gelenkes bestehen, der Arbeitsunfähigkeit bedingte; eine Rückkehr zur Norm bei phlegmonösen Formen wurde nicht oft, Versteifungen und Ankylose nicht gerade selten beobachtet.

Mit der Staungstherapie dagegen erreicht man durch die schon oben erwähnte raschere Beseitigung der entzündlichen Symptome und die frühzeitig ermöglichten Bewegungsübungen eine Verkürzung der Krankheitsdaner und bessere Gelenkfunktionen.

Eine eingehendere Betrachtung der vorliegenden Krankengeschichten bestätigt durchaus diese Beobachtung.

Eine besondere Beachtung verdienen die Krankheitsfälle, die im ganz akuten Stadium in Stauungsbehandlung kamen. Denn gerade für die ganz frischen Fälle will Bier die Stauung in den Vordergrund der Therapie gestellt wissen, da die ersten Tage entscheidend seien für die Gelenkfunktion. Denn hier ist es leicht, die Wirkung der venösen Hyperämie zu entfalten, die Entzündungserscheinungen aufs höchste zu steigern, kurz das zu erreichen, was nach ihm den natürlichen Heilungsvorgang am nachhaltigsten unterstützt. Leider bekommt man verhältnismäßig selten frische Fälle in Behandlung. Das Krankheitsbild der gonorrhoischen Arthritis wird, wenn auch scharf umschrieben, doch noch recht häufig verkannt und unterliegt am meisten der Verwechslung mit dem Gelenk- 
rheumatismus; erst die Erfolglosigkeit der Salicyltherapie weist auf die falsche Diagnose.

So stehen uns nur 13 Fälle zur Verfügung, die innerhalb des 3. bis 6 . Krankheitstages in unsere Behandlung kamen. Darunter waren 7 Handgelenke, 3 Ellenbogen- und 3 Schultergelenke.

Mit Ausnahme von 5 Fällen (3, 4, 5, 12 und 13) handelte es sich um schwere phlegmonöse Formen.

Die Behandlungszeit umfaßte 8 Tage bis 6 Wochen.

Alle 13 Gelenke sind mit unbeschränkter Funktion geheilt.

Was die übrigen 27 Fälle anlangt, so sind 10 Tage bis 21 Wochen verstrichen, bevor dieselben nach anderweitiger, meist erfolgloser Therapie mit Staung behandelt wurden. Die Stauungsbehandlung hat in diesen Fällen um so bessere funktionelle Resultate gezeitigt, je früher mit ihr begonnen wurde. Eine relativ bessere Prognose haben ferner die Gelenke, die noch heftigere Entzündungssymptome zeigen; weil eben ein starker Entzündungsreiz auch ein kräftiges Stauungsödem erreichen läßt.

In einzelnen Fällen haben wir auch nach dem Abklingen der Entzündungserscheinungen die Stauung fortgesetzt, hier im Vertrauen auf die auflösende Wirkung der Hyperämie, um eventuell bindegewebige Verwachsungen - Residuen des lang etablierten Prozesses - durch seröse Durchtränkung und Quellung weicher und dehnbarer zu machen, und durch Massage zur Resorption zu bringen.

Die lange Dauer des Krankheitsprozesses brachte nun da und dort schwere Schädigungen des Gelenkapparates mit sich, die auch für die erst nach Wochen einsetzende Staung irreparabel waren, so besonders im Falle 18, 20, 22, 25, 26 und 30 . Andererseits beweisen gerade wieder diese Fälle die Leistungsfähigkeit der Staubehandlung, indem sie selbst bei sehr vernachiässigten, von anderer Seite aufgegebenen Fällen noch relativ gute Erfolge ermöglicht.

Unter den 27 Fällen waren 12 mal das Handgelenk, 7 mal das Ellenbogengelenk, 4 mal das Schultergelenk und je 1 mal Fuß- und Kniegelenk befallen. Nur zweimal handelte es sich um eine multiple Gelenkerkrankung; einmal war das Hand- und Kniegelenk. im zweiten Fall Knie- und Fußgelenk zu gleicher Zeit erkrankt.

Die Behandlungsdauer dieser Fälle erstreckte sich über einen Zeitraum von 6 Tagen bis 3 Monaten.

Was die funktionellen Resultate anbelangt, so sind 12 Fälle mit voller Funktion ausgeheilt, und zwar 3 Handgelenke, alle 
Zur Beh. d. Arthr. gonorrh. d. großen Gelenke mittels Stauungshyperänie. 53

4 Schultergelenke, 2 Ellenbogengelenke, 1. Fußgelenk und die 4 Gelenke der 2 multiplen Formen.

9 Fälle, und zwar 5 Hand-, 3 Ellenbogen- und 1 Kniegelenk zeigen eine geringe Bewegungsbeschränkung.

Die übrigen 6 Fälle, 4 Hand- und 2 Ellenbogengelenke, haben eine größere Einbuße der Funktion erlitten, jedoch nur ungefähr bis zur Hälfte der normalen.

Ankylosen haben wir nicht erlebt; ein Erfolg der Stauungstherapie, der um so bemerkenswerter ist in Anbetracht der teils sehr schweren, teils sehr vernachlässigten Fälle. Vielleicht verdanken wir einmal der Einführung der Stauungstherapie, wenn unsere 40 Fälle einen Schluß zulassen, daß die Ankylose ans dem Krankheitsbild der gonorrhoischen Arthritis verschwindet.

Bezüglich der genaueren Daten verweise ich auf eine tabellarische Zusammenstellung und auf die Krankengeschichten.

Zum Schluß können wir unsere Erfahrungen in folgende Sätze zusammenfassen:

Die Stauungsbehandlung stellt einen ganz wesentlichen Fortschritt in der Therapie der Arthritis gonorrhoica dar.

Sie zeichnet sich aus durch ihre schmerzlindernde Wirkung.

Der klinische Verlauf der Erkrankung ist unter der Stauung ein verhältnismäßig milder.

Eine Mobilisierung der erkrankten Gelenke ist frühzeitig ermöglicht.

Dadurch ist die Krankheits- und Behandlungsdauer wesentlich verkürzt, die funktionellen Resultate bessere.

Ankylosen sind, soweit unser Material in Frage kommt, nicht beobachtet.

Die Behandlungsmethode ist einfach, technisch nicht schwierig und durch ihre Billigkeit auch dem poliklinischen Kranken zugänglich.

Auf Grund dieser Beobachtungen ist der Staungstherapie eine noch ausgedehntere Verwendung in der Behandlung der Arthritis gonorrhoica einzuräumen.

(Tabellen zu dieser Arbeit siehe nächste Seiten.) 


\begin{tabular}{|c|c|c|c|c|c|c|c|}
\hline $\mathrm{Nr}$. & Name & $\begin{array}{c}\text { Ge- } \\
\text { schlecht }\end{array}$ & Alter & Stand & $\begin{array}{c}\text { Erkranktes } \\
\text { Gelenk }\end{array}$ & $\begin{array}{l}\text { Klinische Er- } \\
\text { krankungs- } \\
\text { form }\end{array}$ & $\begin{array}{l}\text { Eintritt in } \\
\text { die Behand- } \\
\text { lung }\end{array}$ \\
\hline 1. & M. K. & w. & $17 \mathrm{~J}$. & $\begin{array}{r}\text { Dienst- } \\
\text { mädchen }\end{array}$ & R. Handge- & $\begin{array}{c}\text { Schwere } \\
\text { phlegmonöse } \\
\text { Arthritis }\end{array}$ & $\underset{\text { heitstag }}{\text { Am } 6 . \text { Krank- }}$ \\
\hline 2. & $\mathrm{~T}$. & w. & 25. J. & & R. Handge- & $\begin{array}{c}\text { Sehr schwere } \\
\text { phlegmonöse } \\
\text { Form }\end{array}$ & 3. Tag \\
\hline 3. & F. T. & m. & $30 \mathrm{~J}$. & Monteur & L. Handge- & $\begin{array}{l}\text { Leichte Ar- } \\
\text { thritis sero- } \\
\text { fibrinosa }\end{array}$ & 3. Tag \\
\hline 4. & K. B. & m. & $29 \mathrm{~J}$. & Kellner & $\begin{array}{l}\text { R. Handge- } \\
\text { lenk }\end{array}$ & $\begin{array}{c}\text { Leichte } \\
\text { phlegmonöse } \\
\text { Form }\end{array}$ & 6. Tag \\
\hline 5. & P. & w. & $18 \mathrm{~J}$. & $\begin{array}{l}\text { Nähte. } \\
\text { rin }\end{array}$ & $\begin{array}{l}\text { L. Handge- } \\
\text { lenk }\end{array}$ & $\begin{array}{c}\text { Leichte } \\
\text { phlegmonöse } \\
\text { Form }\end{array}$ & 6. Tag \\
\hline 6. & F. Sb. & m. & $26 \mathrm{~J}$. & $\begin{array}{l}\text { Büchsen- } \\
\text { macher }\end{array}$ & L. Handge- & $\begin{array}{c}\text { Phlegmonöse } \\
\text { Form }\end{array}$ & 6. Tag \\
\hline 7. & E. E. & w. & $26 \mathrm{~J}$ & $\begin{array}{l}\text { Melke- } \\
\text { frau }\end{array}$ & R. Handge- & $\begin{array}{l}\text { Phlegmonöse } \\
\text { Form }\end{array}$ & 5. Tag \\
\hline 8. & H. K. & w. & $16 \mathrm{~J}$ & & $\begin{array}{l}\text { R. Ellbogen- } \\
\text { gelenk }\end{array}$ & $\begin{array}{l}\text { Phlegmonöse } \\
\text { Form }\end{array}$ & 4. Tag \\
\hline 9. & M. B. & w. & $18 \mathrm{~J}$ & $\begin{array}{l}\text { Dienst- } \\
\text { mädchen }\end{array}$ & $\begin{array}{l}\text { R. Ellbogen- } \\
\text { gelenk }\end{array}$ & $\begin{array}{l}\text { Phlegmonöse } \\
\text { Form }\end{array}$ & 2. Tag \\
\hline 10. & Sch. & w. & $20 \mathrm{~J}$ & $\begin{array}{l}\text { Nähte- } \\
\text { rin }\end{array}$ & $\begin{array}{l}\text { L. Ellbogen- } \\
\text { gelenk }\end{array}$ & $\begin{array}{c}\text { Sehr schwere } \\
\text { phlegmonöse } \\
\text { Form }\end{array}$ & 6. Tag \\
\hline 11. & R. & w. & $45 \mathrm{~J}$ & & $\begin{array}{l}\text { R. Schulter- } \\
\text { gelenk }\end{array}$ & $\begin{array}{l}\text { Phlegmonöse } \\
\text { Form }\end{array}$ & 3. Tag \\
\hline 12. & H. K. & w. & $28 \mathrm{~J}$. & $\begin{array}{l}\text { Dienst- } \\
\text { mädchen }\end{array}$ & $\begin{array}{l}\text { L. Schulter- } \\
\text { gelenk }\end{array}$ & $\begin{array}{l}\text { Arthritis sero- } \\
\text { fibrinosa }\end{array}$ & 4. Tag \\
\hline 13. & W.W. & m. & $35 \mathrm{~J}$. & $\begin{array}{l}\text { Kauf- } \\
\text { mann }\end{array}$ & $\begin{array}{l}\text { L. Schulter- } \\
\text { gelenk }\end{array}$ & $\begin{array}{c}\text { Arthritis } \\
\text { serofibrinosa }\end{array}$ & 3. Tag \\
\hline
\end{tabular}


Zur Beh. d. Arthr. gonorrh. d. großen Gelenke mittels Stauungshyperämie. 55

\begin{tabular}{|c|c|c|c|c|}
\hline $\begin{array}{l}\text { Dauer der } \\
\text { Einzel- } \\
\text { stauung }\end{array}$ & $\begin{array}{l}\text { Dauer der } \\
\text { Staube- } \\
\text { handlung }\end{array}$ & $\begin{array}{c}\text { Weitere } \\
\text { therapeu- } \\
\text { tische Maß- } \\
\text { nahmen }\end{array}$ & $\begin{array}{c}\text { Gesamt- } \\
\text { Dauer der } \\
\text { Behand- } \\
\text { lung }\end{array}$ & Endresultat \\
\hline 22 Stunden & 4 Wochen & Massage & 1. Monat & $\begin{array}{l}\text { Gelenkkonturen normal. } \\
\text { Unbeschränkte Funktion des } \\
\text { Handgelenks und der Finger. }\end{array}$ \\
\hline 22 Stunden & 8 Tage & Massage & 8 Tage & Desgl. \\
\hline 22 Stunden & 5 Tage & Massage & 7 Tage & Desgl. \\
\hline 22 Stunden & 8 Tage & Massage & 12 Tage & Desg!. \\
\hline 22 Stunden & 6 Tage & Massage & 8 Tage & Desgl. \\
\hline 22 Stunden & 8 Tage & Massage & 12 Tage & DesgI. \\
\hline 22 Stunden & 18 Tage & Massage & 18 Tage & Desgl. \\
\hline $\begin{array}{l}\text { 7-12 Stdn. } \\
\text { u. weniger }\end{array}$ & 6 Wochen & Massage & 6 Wochen & $\begin{array}{l}\text { Gelenkkonturen normal. } \\
\text { Vollkommene Funktion nach } \\
\text { allen Richtungen hin. }\end{array}$ \\
\hline $\begin{array}{l}\text { Zuerst } 4-5 \\
\text { Stunden, } \\
\text { später } 22 \\
\text { Stunden }\end{array}$ & $41 / 2$ Woch. & Massage & $51 / 2$ Woch. & $\begin{array}{l}\text { Funktion wie links. (Beider- } \\
\text { seits seit der Kindheit infolge } \\
\text { Rachitis ca. 100 Streckbe- } \\
\text { hinderung.) }\end{array}$ \\
\hline 22 Stunden & $\begin{array}{l}4 \text { Wochen, } \\
1 \text { Woche } \\
\text { unterbroch. }\end{array}$ & Massage & $5^{1 / 2}$ Woch. & $\begin{array}{l}\text { Gelenkkonturen unverän- } \\
\text { dert. Volle Funktion. }\end{array}$ \\
\hline 22 Stunden & 8 Tage & Massage & 8 Tage & $\begin{array}{l}\text { Gelenkkonturen } \\
\text { Volle Funktion. }\end{array}$ \\
\hline 22 Stunden & $31 / 2$ Woch. & Massage & - & Desgl. \\
\hline 22 Stunden & 8 Tage & Massage & 14 Tage & Desgl. \\
\hline
\end{tabular}




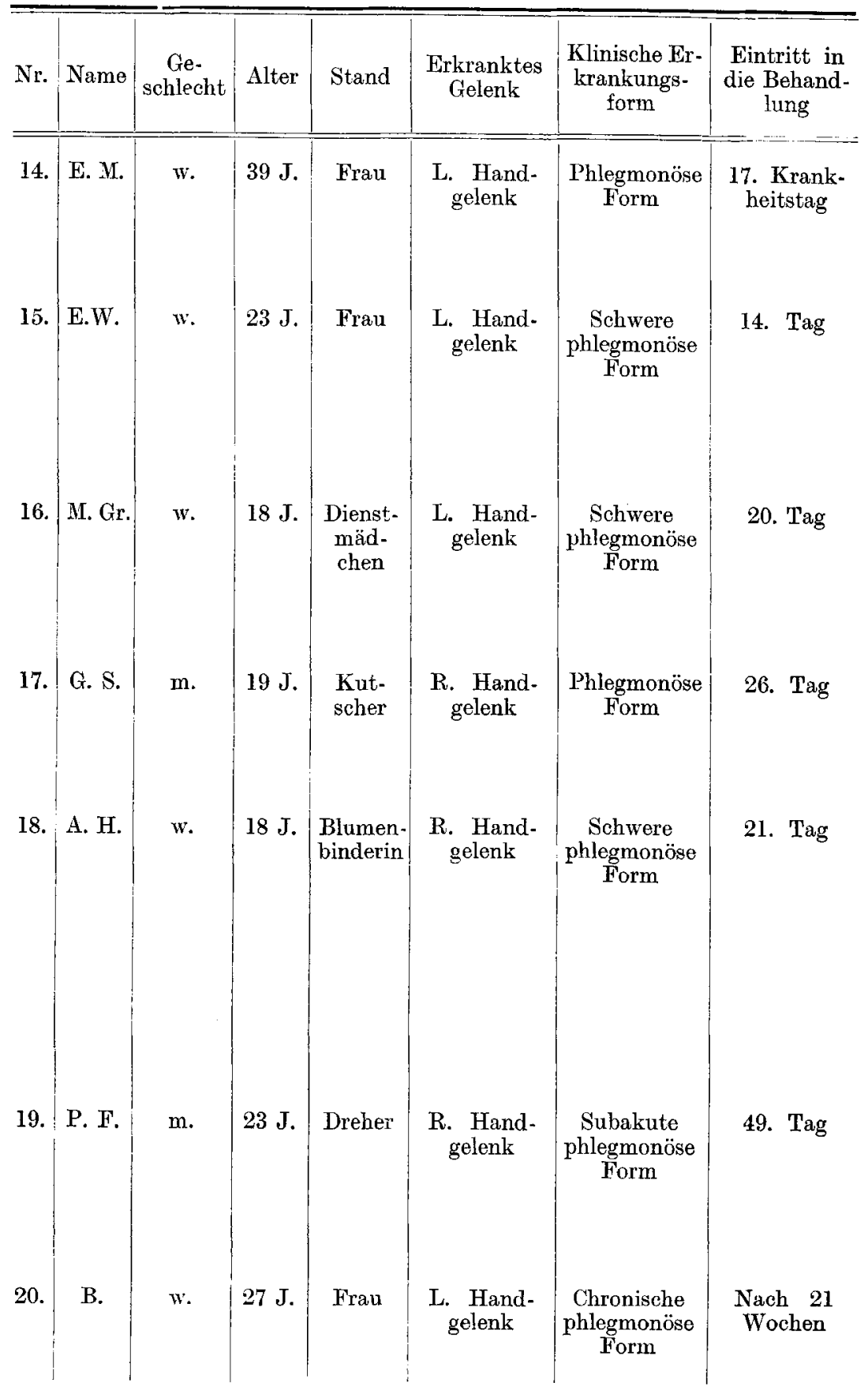


Zur Beh. d. Arthr. gonorrh. d. großen Gelenke mittels Stauungshyperämie. 57

\begin{tabular}{|c|c|c|c|c|}
\hline $\begin{array}{l}\text { Dauer der } \\
\text { Einzel- } \\
\text { stauung }\end{array}$ & $\begin{array}{l}\text { Dauer der } \\
\text { Staube- } \\
\text { handlung }\end{array}$ & $\begin{array}{c}\text { Weitere } \\
\text { therapeu- } \\
\text { tische Maß- } \\
\text { nahmen }\end{array}$ & $\begin{array}{c}\text { Gesamt- } \\
\text { Dauer der } \\
\text { Behand- } \\
\text { lung }\end{array}$ & Endresultat \\
\hline 8 Stunden & $\begin{array}{l}\text { ca. } 10 \\
\text { Wochen }\end{array}$ & 一 & 10 Wochen & $\begin{array}{l}\text { Gelenkkonturen normal. } \\
\text { Bewegungen im Handgelenk } \\
\text { frei. Der 2. und } 5 \text {, Finger } \\
\text { bleibt beim Beugeversuch ca. } \\
5 \mathrm{~cm} \text { vom Handteller entfernt. }\end{array}$ \\
\hline 12 Stunden & 5 Wochen & - & 5 Wochen & $\begin{array}{l}\text { Konturen normal. Mo- } \\
\text { torische Kraft herabsesetzt. } \\
\text { Krepitation im Radiocarpal- } \\
\text { gelenk. Handgelenksbewegun- } \\
\text { gen bis auf } 5^{0} \text { Streckbehinde- } \\
\text { rung vollkommen frei. Finger- } \\
\text { bewegungen unbeschränkt. }\end{array}$ \\
\hline 12 Stunden & ca. 3 Mon. & - & 3 Monate & $\begin{array}{l}\text { Zeitweise Schmerzen. Ge- } \\
\text { lenkkonturen normal. Kre- } \\
\text { pitation. Handgelenksbe- } \\
\text { wegungen bis auf } 10^{\circ} \text { Differenz } \\
\text { in Beugung und Streckung wie } \\
\text { rechts. Finger frei. }\end{array}$ \\
\hline 12 Stunden & 8 Wochen & - & - & $\begin{array}{l}\text { Motorische Schwäche der } \\
\text { Hand. Oberarmmuskulatur } \\
\text { atrophisch. Gelenkkonturen } \\
\text { normal. Unbeschränkte Funk- } \\
\text { tion von Hand und Finger. }\end{array}$ \\
\hline 22 Stunden & $5^{1 / 2}$ Woch. & $\begin{array}{l}8 \text { Tage } \\
\text { Handsaug- } \\
\text { glas; Mas- } \\
\text { sage; heiße } \\
\text { Bäder }\end{array}$ & 13 Wochen & 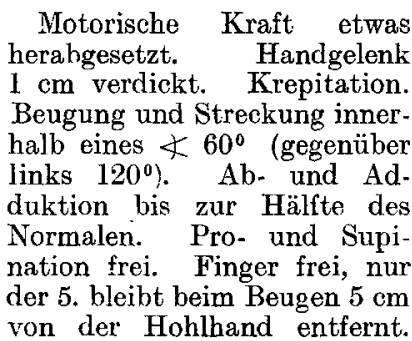 \\
\hline 22 Stunden & 6 Wochen & Massage & 7 Wochen & $\begin{array}{l}\text { Handgelenk } 1 / 2 \mathrm{~cm} \text { verdickt. } \\
\text { Motorische Kraft herabgesetzt. } \\
\text { Fingerbewegungen frei. Hand- } \\
\text { gelenk bis auf eine kleine }\left(10^{\circ}\right) \\
\text { Differenz im Beugen und } \\
\text { Strecken ganz beweglich. }\end{array}$ \\
\hline 22 Stunden & 21 Tage & Massage & 4 Wochen & $\begin{array}{l}\text { Beugung und Streckung der } \\
\text { Hand innerh. } 60-76^{\circ} \text {, Abduk- } \\
\text { tion ganz behindert, Adduk- } \\
\text { tion bis zur Hälfte. Finger frei. }\end{array}$ \\
\hline
\end{tabular}




\begin{tabular}{|c|c|c|c|c|c|c|c|}
\hline $\mathrm{Nr}$. & Name & $\begin{array}{c}\text { Ge- } \\
\text { schlecht }\end{array}$ & Alter & Stand & $\begin{array}{c}\text { Erkranktes } \\
\text { Gelenk }\end{array}$ & $\begin{array}{l}\text { Klinische } \\
\text { Erkrankungs- } \\
\text { form }\end{array}$ & $\begin{array}{l}\text { Eintritt in } \\
\text { die Behand- } \\
\text { lung }\end{array}$ \\
\hline 21. & H.Sch. & w. & $19 \mathrm{~J}$. & $\begin{array}{l}\text { Dienst- } \\
\text { mäd- } \\
\text { chen }\end{array}$ & $\begin{array}{l}\text { R. Hand- } \\
\text { gelenk }\end{array}$ & Arthralgie & 12. Tag \\
\hline 22. & M. K. & w. & $18 \mathrm{~J}$ & $\begin{array}{l}\text { Dienst- } \\
\text { mäd- } \\
\text { chen }\end{array}$ & $\begin{array}{l}\text { R. Hand- } \\
\text { gelenk }\end{array}$ & $\begin{array}{c}\text { Chronische } \\
\text { phlegmonöse } \\
\text { Form }\end{array}$ & $\begin{array}{l}\text { Nach } 9 \\
\text { Wochen }\end{array}$ \\
\hline 23. & E. M. & w. & $18 \mathrm{~J}$. & $\begin{array}{l}\text { Dienst- } \\
\text { mäd. } \\
\text { chen }\end{array}$ & $\begin{array}{c}\text { R. Hand- } \\
\text { gelenk }\end{array}$ & $\begin{array}{c}\text { Chronische } \\
\text { phlegmonöse } \\
\text { Form }\end{array}$ & $\begin{array}{l}\text { Nach } 11 \\
\text { Wochen }\end{array}$ \\
\hline 24. & A. St. & w. & $24 \mathrm{~J}$ & & $\begin{array}{l}\text { L. Hand- } \\
\text { gelenk }\end{array}$ & $\begin{array}{l}\text { Phlegmonöse } \\
\text { Form }\end{array}$ & Am 16. Tage \\
\hline 25. & F. S. & $\mathrm{m}$. & $25 \mathrm{~J}$. & Arbeiter & $\begin{array}{l}\text { R. Hand- } \\
\text { gelenk }\end{array}$ & $\begin{array}{l}\text { Sehr schwere } \\
\text { phlegmonöse } \\
\text { Form }\end{array}$ & 33. Tag \\
\hline 26. & W. B. & $\mathrm{m}$. & $20 \mathrm{~J}$ & $\begin{array}{l}\text { Kauf- } \\
\text { mann }\end{array}$ & $\begin{array}{l}\text { R. Ellbogen- } \\
\text { gelenk }\end{array}$ & $\begin{array}{c}\text { Phlegmonöse } \\
\text { Form }\end{array}$ & $\begin{array}{l}\text { Nach } 5 \\
\text { Wochen }\end{array}$ \\
\hline 27. & E. W. & w. & $29 \mathrm{~J}$. & Frau & $\begin{array}{l}\text { R. Ellbogen- } \\
\text { gelenk }\end{array}$ & $\begin{array}{l}\text { Phlegmonöse } \\
\text { Form }\end{array}$ & Am 17. Tag \\
\hline 28. & K. G. & w. & $26 \mathrm{~J}$. & $\begin{array}{l}\text { Expe- } \\
\text { dientin }\end{array}$ & $\begin{array}{l}\text { R. Ellbogen- } \\
\text { gelenk }\end{array}$ & $\begin{array}{c}\text { Sehr schwere } \\
\text { phlegmonöse } \\
\text { Form }\end{array}$ & 25. Tag \\
\hline 29 . & M. & $\mathrm{m}$. & $21 \mathrm{~J}$ & Arbeiter & $\begin{array}{l}\text { R. Ellbogen- } \\
\text { gelenk }\end{array}$ & $\begin{array}{c}\text { Phlegmonöse } \\
\text { Form }\end{array}$ & 10. Tag \\
\hline
\end{tabular}


Zur Beh. d. Arthr. gonorrh. d. großen Gelenke mittels Stauungshyperämie. 59

\begin{tabular}{|c|c|c|c|c|}
\hline $\begin{array}{l}\text { Dauer der } \\
\text { Einzel- } \\
\text { stauung }\end{array}$ & $\begin{array}{l}\text { Dauer der } \\
\text { Staube- } \\
\text { hardlung }\end{array}$ & $\begin{array}{c}\text { Weitere } \\
\text { therapen- } \\
\text { tische Maß- } \\
\text { nahmen }\end{array}$ & $\begin{array}{l}\text { Gesamt- } \\
\text { Dauer der } \\
\text { Behand- } \\
\text { lung }\end{array}$ & Endresultat \\
\hline 22 Stunden & 5 Tage & Massage & 6 Tage & $\begin{array}{l}\text { Normale Gelenkkonturen. } \\
\text { Unbeschränkte Funktion. }\end{array}$ \\
\hline 22 Stunden & 18 Tage & Massage & 18 Tage & $\begin{array}{l}\text { Beugung und Streckung der } \\
\text { Hand innerhalb } 70^{\circ} \text {; Ad- } \\
\text { duktion etwas behindert. } \\
\text { Sonstige Bewegungen frei. } \\
\text { Finger unbeschränkt. }\end{array}$ \\
\hline 22 Stunden & 6 Tage & Massage & 6 Tage & $\begin{array}{l}\text { Bewegungen der Hand bis } \\
\text { auf } 100 \text { Extensionsbehinderung } \\
\text { frei. Finger beweglich. }\end{array}$ \\
\hline 22 Stunden & 10 Tage & Massage & 14 Tage & $\begin{array}{l}\text { Gelenkkonturen normal. } \\
\text { Hand und Finger vollkommen } \\
\text { frei beweglich. }\end{array}$ \\
\hline 22 Stunden & 18 Tage & Massage & $\begin{array}{c}7-8 \\
\text { Wochen }\end{array}$ & $\begin{array}{l}\text { Motorische Kraft der Hand } \\
\text { herabgesetzt. Handgelenk } \\
1 \text { cm verdickt. Hand Spur } \\
\text { radialwärts verschoben. Beu- } \\
\text { gung und Streckung inner- } \\
\text { halb } 80^{\circ} \text { (gegen } 120^{\circ} \text { links). } \\
\text { Ab- und Adduktion bis zur } \\
\text { Hälfte des Normalen. Pro- und } \\
\text { Supination frei. Finger un- } \\
\text { beschränkt. }\end{array}$ \\
\hline 1-4 Stunden & $\begin{array}{l}\text { ca. } 3 \text { Mo- } \\
\text { naten }\end{array}$ & Massage & 3 Monate & $\begin{array}{l}\text { Motorische Kraft des Arms } \\
\text { wesentlich vermindert. Beu- } \\
\text { gung im Ellbogengel. } 75^{\circ} \text {, Strek- } \\
\text { kung } 150^{\circ} \text {; Pronation und } \\
\text { Supination etwas behindert. }\end{array}$ \\
\hline 6-12 Stdn. & 6 Wochen & Massage & 8 Wochen & $\begin{array}{l}\text { Normale Gelenkkonturen. } \\
\text { Krepitation. Alle Bewegungen } \\
\text { bis auf geringe Streckbe- } \\
\text { hinderung }\left(\mathbf{1 0}^{\circ}\right) \text { vollkommen } \\
\text { frei. }\end{array}$ \\
\hline 22 Stunden & 25 'Tage & Massage & 4 Wochen & $\begin{array}{l}\text { Gelenkkonturen normal. } \\
\text { Alle Bewegungen wie links, } \\
\text { nur geringe Extensionsbe- } \\
\left.\text { hinderung (ca } 10^{\circ}\right)\end{array}$ \\
\hline $18-22$ Stdn. & 10 Tage & $\begin{array}{l}\text { Massage u. } \\
\text { Heißluft }\end{array}$ & 18 Tage & $\begin{array}{l}\text { Normale Gelenkkonturen. } \\
\text { Volle Funktion. }\end{array}$ \\
\hline
\end{tabular}




\begin{tabular}{|c|c|c|c|c|c|c|c|}
\hline Nr. & Name & $\begin{array}{c}\text { Ge- } \\
\text { schlecht }\end{array}$ & Alter & Stand & $\begin{array}{c}\text { Erkranktes } \\
\text { Gelenk }\end{array}$ & $\begin{array}{l}\text { Klinische Er- } \\
\text { krankungs- } \\
\text { form }\end{array}$ & $\begin{array}{l}\text { Eintritt in } \\
\text { die Behand- } \\
\text { lung }\end{array}$ \\
\hline 30. & W. F. & $\mathrm{m}$. & $30 \mathrm{~J}$. & $\begin{array}{c}\text { Gerichts- } \\
\text { diener }\end{array}$ & $\begin{array}{l}\text { R. Ellbogen- } \\
\text { gelenk }\end{array}$ & $\begin{array}{c}\text { Chronische } \\
\text { phlegmonöse } \\
\text { Form }\end{array}$ & $\begin{array}{l}\text { Nach } 10 \\
\text { Wochen }\end{array}$ \\
\hline 31. & H. Fr. & $\mathrm{m}$. & $31 \mathrm{~J}$. & $\begin{array}{l}\text { Kauf- } \\
\text { mann }\end{array}$ & $\begin{array}{l}\text { L. Ellbogen- } \\
\text { gelenk }\end{array}$ & $\begin{array}{c}\text { Arthritis se- } \\
\text { rofibrinosa } \\
\text { gon. }\end{array}$ & $\begin{array}{c}\text { Nach } 10 \\
\text { Tagen }\end{array}$ \\
\hline 32. & H. & w. & - & Frau & $\begin{array}{l}\text { R. Ellbogen- } \\
\text { gelenk }\end{array}$ & $\begin{array}{c}\text { Phlegmonöse } \\
\text { Form }\end{array}$ & $\begin{array}{c}\text { Nach ca. } 2 \\
\text { Monaten }\end{array}$ \\
\hline 33. & F. T. & w. & $27 \mathrm{~J}$. & Frau & $\begin{array}{l}\text { R. Schulter- } \\
\text { gelenk }\end{array}$ & $\begin{array}{c}\text { Arthritis se- } \\
\text { rofibrinosa } \\
\text { gon. }\end{array}$ & 18. Tag \\
\hline 34. & S. & $w$. & $19 \mathrm{~J}$. & $\begin{array}{l}\text { Konto- } \\
\text { ristin }\end{array}$ & $\begin{array}{l}\text { L. Schulter- } \\
\text { gelenk }\end{array}$ & $\begin{array}{l}\text { Arthritis se- } \\
\text { rcfibrinosa } \\
\text { gon. }\end{array}$ & 14. Tag \\
\hline 35. & P. & w. & $37 \mathrm{~J}$. & $\begin{array}{c}\text { Ar- } \\
\text { beiterin }\end{array}$ & $\begin{array}{l}\text { L. Schulter- } \\
\text { gelenk }\end{array}$ & $\begin{array}{l}\text { Arthritis se- } \\
\text { rofibrinosa } \\
\text { gon. }\end{array}$ & 45. Tag \\
\hline 36. & B. & $w$. & $42 \mathrm{~J}$. & - & $\begin{array}{l}\text { L. Schulter- } \\
\text { gelenk }\end{array}$ & $\begin{array}{l}\text { Arthritis se- } \\
\text { rofibrinosa } \\
\text { gon. }\end{array}$ & 30. Tag \\
\hline 37. & $\begin{array}{l}\text { M. } \\
\text { Schr. }\end{array}$ & w. & $21 \mathrm{~J}$. & $\begin{array}{c}\text { Ver- } \\
\text { käuferin }\end{array}$ & $\begin{array}{l}\text { L. Kniege- } \\
\text { lenk }\end{array}$ & $\begin{array}{l}\text { Arthritis se- } \\
\text { rofibrinosa } \\
\text { gon. }\end{array}$ & $\begin{array}{l}\text { Nach ca. } 3 \\
\text { Monaten }\end{array}$ \\
\hline 38. & P. & w. & $27 \mathrm{~J}$. & Frau & $\begin{array}{l}\text { R. Fußge- } \\
\text { lenk }\end{array}$ & $\begin{array}{c}\text { Phlegmonöse } \\
\text { Form }\end{array}$ & $\begin{array}{c}\text { Nach } 11 \\
\text { Tagen }\end{array}$ \\
\hline 39. & K. W. & $\mathrm{m}$. & $23 \mathrm{~J}$. & Kellner & $\begin{array}{l}\text { R. Hand- } \\
\text { gelenk u. r. } \\
\text { Kniegelenk }\end{array}$ & $\begin{array}{l}\text { Arthritis se- } \\
\text { rofibrinosa } \\
\text { manus et } \\
\text { genus }\end{array}$ & $?$ \\
\hline 40. & B. & w. & $25 \mathrm{~J}$ & Frau & $\begin{array}{l}\text { L. Knie- und } \\
\text { 1. Fußgelenk }\end{array}$ & $\begin{array}{l}\text { Fußgelenk: } \\
\text { phleg. Form. } \\
\text { Knieg.: Arthr. } \\
\text { serofibrinosa }\end{array}$ & $\begin{array}{c}\text { Nach } 71 / 2 \\
\text { Wochen }\end{array}$ \\
\hline
\end{tabular}


Zur Beh. d. Arthr. gonorrh. d. großen Gelenke mittels Staunngshyperämie. 61

\begin{tabular}{|c|c|c|c|c|}
\hline $\begin{array}{l}\text { Dauer der } \\
\text { Einzel- } \\
\text { stauung }\end{array}$ & $\begin{array}{l}\text { Dauer der } \\
\text { Staube- } \\
\text { handlung }\end{array}$ & $\begin{array}{c}\text { Weitere } \\
\text { therapeu- } \\
\text { tische Maß- } \\
\text { nahmen }\end{array}$ & $\begin{array}{c}\text { Gesamt- } \\
\text { Dauer der } \\
\text { Behand- } \\
\text { lung }\end{array}$ & Endresultat \\
\hline 22 Stunden & 14 Tage & Massage & $61 / 2$ Woch. & $\begin{array}{l}\text { Motorische Kraft des rechten } \\
\text { Armes herabgesetzt. Ober- } \\
\text { armmuskulatur atrophisch. } \\
\text { Ellbogengelenk } 2 \text { cm verdickt. } \\
\text { Krepitation. Beugung } 70^{\circ} \text {, } \\
\text { Streckung bis } 170^{\circ} \text {. Supination } \\
\text { etwas beschränkt. Pronat. frei. }\end{array}$ \\
\hline 22 Stunden & 8 Tage & Massage & 8 Tage & $\begin{array}{l}\text { Normale Gelenkkonturen. } \\
\text { Volle Funktion. }\end{array}$ \\
\hline 22 Stunden & 5 Wochen & Massage & 30 Tage & $\begin{array}{l}\text { Normale Gelenkkonturen. } \\
\text { Beugung, Pro- und Supination } \\
\text { frei. Streckbehinderung ca. } 20^{\circ} \text {. }\end{array}$ \\
\hline 1-12 Stdn. & $?$ & - & $\begin{array}{c}\text { ca. } 9 \\
\text { Wochen }\end{array}$ & $\begin{array}{l}\text { Normale Gelenkkonturen. } \\
\text { Vollkommene Funktion. }\end{array}$ \\
\hline 1 Stunde & 14 Tage & - & 16 Tage & Desgl. \\
\hline 22 Stunden & 25 Tage & Massage & 4 Wochen & Desgl. \\
\hline 22 Stunden & 7 Wochen & Massage & 8 Wochen & Desgl. \\
\hline 22 Stunden & $\begin{array}{c}3-4 \\
\text { Wochen }\end{array}$ & Massage & 1 Monat & $\begin{array}{l}\text { Oberschenkelmuskulatur et- } \\
\text { was atrophisch. Beugung } 60^{\circ} \text {, } \\
\text { Streckung unbehindert. }\end{array}$ \\
\hline 22 Stunden & 3 Wochen & $\begin{array}{c}\text { Heißluft } \\
\text { u. Massage; } \\
\text { heiße Bä- } \\
\text { der }\end{array}$ & $81 / 2$ Woch. & $\begin{array}{l}\text { R. Fußgelenk } 1 \text { cm dicker } \\
\text { wie links; leicht hinkender } \\
\text { Gang. Bewegungen im Fußge- } \\
\text { lenk ebenso ausgiebig wie links. }\end{array}$ \\
\hline 22 Stunden & $\begin{array}{l}\text { Hand: } 14 \\
\text { Tage;Knie: } \\
21 \text { Tage }\end{array}$ & $\begin{array}{c}\text { Aktive und } \\
\text { passive Be- } \\
\text { wegungen }\end{array}$ & 7 Wochen & $\begin{array}{l}\text { Gelenkkonturen: Handge- } \\
\text { lenk und Kniegelenk normal. } \\
\text { Volle Funktion. }\end{array}$ \\
\hline 22 Stunden & 4 Wochen & $\left|\begin{array}{c}\text { Heißluft } \\
\text { u. Massage; } \\
\text { heiße Bä- } \\
\text { der }\end{array}\right|$ & $\begin{array}{c}\text { ca. } 3 \mathrm{Mo} \text {. } \\
\text { nate }\end{array}$ & $\begin{array}{l}\text { Hinkender Gang. Geienk- } \\
\text { konturen: Knie normal, Fuß } \\
\text { etwas verdickt. Funktion: Fuß- } \\
\text { u. Kniegelenk unbeschränkt. }\end{array}$ \\
\hline
\end{tabular}




\section{Krankengeschichten.}

1. M. K., 17 Jahre alt, Dienstmädchen. Früher stets gesund. Seit 20. V. 1907 eitrigen AusfluB. Am 28. V. Anschwellung des rechten Handgelenkes, starke Rötung. Unbeweglichkeit von Hand und Finger. Schlechtes Allgemeinbefinden, Schlaflosigkeit. Feuchte Umschläge. Mitella. Am 3. VI. 1907 Poliklinik.

Status: Das rechte Handgelenk ist diffus polsterartig geschwollen, die Schwellung dehnt sich aus über die Finger und den Unterarm entlang bis hinauf zum Ellbogen. Die Haut ist stark entzündlich gerötet, ödematös und gespannt.

Maße: oberhalb der Proc. styloidei rechts $22 \mathrm{~cm}$, links $16 \mathrm{~cm}$, unterhalb der Proc. styloidei in der Handgelenksfurche $22 \mathrm{~cm}, 1.17 \mathrm{~cm}$, über der größten Breite der Hand rechts $23 \mathrm{~cm}$, links $18 \mathrm{~cm}$.

Das Handgelenk ist in geringer Beugestellung völlig fixiert. Die Finger stehen in Streckstellung fest. Bewegungen aktiv und passiv unmöglich. Spontan äußerste Schmerzhaftigkeit. Temp. 38,2. Schwere phlegmonöse Form. Ab 4.VI. 190722 stündige Stauung. Heißes feuriges Ödem. Schmerzen lassen sofort nach. Pat. schläft die ganze Nacht. 8. VI. Schwellung und Rötung zurückgegangen. Unter der Binde beschwerdefrei. Fingerbewegung aktiv schon ziemlich ergiebig, passive Bewegungen im Handgelenk ohne Schmerzen leidlich ausführbar, aktiv noch nicht. 20. VI. Nach 2 stündiger Suspension Schwellung nur noch gering. Rechts 17, 18, $19 \mathrm{~cm}$; links 16, $17,18 \mathrm{~cm}$. Die Haut blaßt ab und zeigt starke Runzelung. Bewegungen der Finger fast in normaler Ausdehnung möglich, des Handgelenks aktiv innerhalb einem $\measuredangle$ von $60^{\circ}$, Pro- und Supination frei, $\mathrm{Ab}$ - und Adduktion bis zur Hälfte des Normalen, passive Bewegungen noch ausgiebiger.

Stauung bis zum 5. VII. - 4 Wochen - Gelenkkonturen normal. Zeitweise noch Schmerzempfindungen im Handgelenk bei forcierten Bewegungen. Volle Funktion von Hand und Finger. Nachuntersuchung am 20. IX. 1907. Vollkommen normales Gelenk.

2. T., Frau, 25 J. alt. Früher stets gesund. Am 25. VII. gonorrhoische Infektion. 28. VII. 1907 erwacht Pat. mit sehr starken Schmerzen in allen Gelenken, besonders das linke Handgelenk ist äuBerst schmerzhaft geschwollen und gerötet. Am 30. VII. Überweisung zur Klinik.

Status: Das linke Handgelenk ist sehr stark geschwollen und gerötet. Handrücken, Finger und Unterarm sind gleichfalls von ödematöser Schwellung und Rötung befallen. Die Hand steht in leichter Hyperextension, Finger in Krallenstellung, Spur von Bewegung der Finger, dagegen im Handgelenk aktiv und passiv keine Bewegung möglich, ganz enorme Schmerzhaftigkeit. Temp. 38,8. Schwere phlegmonöse Form.

Ab 30. VII. 22 stündige Stauung. 31. VII. Temp.-Abfall 37,1. Nacht gut geschlafen. Másige Bewegungen im Handgelenk aktiv ausführbar und schmerzfrei. 31. VII.-4. VIII. Rasche Besserung. Ab 5. VIII. nur noch 12 stündige Stauung. 7. VIII. Nach 8 Tagen entlassen mit geringem Ödem des Handrückens und ganz normaler Funktion von Hand und Finger.

3. Fritz T., 30 J. alt. Sonst gesund, Tripper. Am 28. VII. sehr heftige Schmerzen in der linken Hand, Schwellung, Unbeweglichkeit der Hand und Finger. Am 30. VII. 1907 Poliklinik. 
Zur Beh. d. Arthr. gonorrh. d. großen Gelenke mittels Stauungshyperämie. 63

Status: Linkes Handgelenk mäBig geschwollen, deutlicher ErguB im Gelenk. Paraartikuläre Weichteile nur mäßig befallen. Bewegungen von Hand und Finger schmerzhaft doch leidlich ausführbar. Arthritis gon. serofibrinosa.

Ab 28.VII. 22 stündige Stauung wird gut ertragen, wirkt rasch schmerzlindernd, Unter der Binde können Bewegungen aktiv und passiv fast in vollem Umfang ausgeführt werden. Nach 5 tägiger Stauung ist Pat. in vollem Gebrauche der Hand. 5. VIII. mit normalem Gelenk und voller Funktion entlassen.

4. B. K., Kellner, 29 J. alt. Sonst gesund. Vor 7 Jahren Tripper. Vor 1 Jahr Rezidiv, seitdem nie ganz geheilt, Fäden im Urin. Seit 15. IX. 1907 Schmerzen im rechten Handgelenk und dem rechten Daumen, starke Schwellung und Rötung. Da unter Umschlägen keine Besserung eintritt, Poliklinik.

Status 20. IX. 1907: Rechte Handgelenksgegend geschwollen, Schwellung erstreckt sich über dem Handrücken entlang bis zu den Köpfchen der Metacarpi; Handteller verstrichen. Im Gelenk deutlicher Erguß. Die Strecksehnen des Daumens sind besonders befallen und auf Druck und bei Bewegungen äuBerst schmerzhaft. Maße: Rechts 19, 19, 23 gegen 17, 17, 21,5 links. Bewegungen sind beschränkt. Beugung $20^{\circ}$, Streckung $10^{\circ}$.

Abduktion etwas behindert. Adduktion möglich; Pro- und Supination frei. Passive Bewegungen etwas ausgiebiger, doch sehr schmerzhaft.

$A b$ 20. IX. 22 stündige Stauung. Sehr heißes starkes Ödem, Schmerzen lassen nach. 25. IX. Rasche Besserung. Schwellung fast vollstandig geschwunden, Strecksehnen des Daumens noch infiltriert und schmerzhaft. 28. IX. Bewegungen aktiv und passiv frei, noch geringe Beschwerden bei Bewegungen des Daumens. Stauung fortgelassen, Massage bis zum 1. X. 1. X. Geheilt mit normaler Gelenkfunktion und arbeitsfähig entlassen.

5. P., 18 J. alt, Nähterin. Seit längerer Zeit Tripper. Am 15. IX. 1907 Partus. Puerperium fieberhaft. In der Nacht vom 25./26. IX. plötzlich sehr starke Schmerzen im linken Handgelenk, Schwellung und Rötung. 27. IX. Poliklinik.

Status: Linkes Handgelenk sehr stark geschwollen, in der Gelenkkapsel deutlicher praller ErguB nachweisbar. Hand steht in Volarflexion und ulnarer Abduktion. Paraartikuläre Weichteile sind wenig beteiligt. UmfangsmaBe: Links $2 \mathrm{~cm}$ mehr wie rechts. Alle Bewegungen im Handgelenk und der Finger wegen enormer Schmerzhaftigkeit behindert. Starke Empfindlichkeit bei Druck auf die beiden Styloidei.

27. IX. 22 stündige Stauung. Sehr starkes Ödem, Schmerzen sistieren sofort; unter der Binde aktive und passive Bewegungen möglich. 29. IX. Rasche Besserung. Schwellung und Rötung nur gering, Kapselerguß ebenfalls zurückgegangen. 1. X. Erguß nicht mehr nachweisbar. Ohne Schmerzen. 3. X. Stauung fort. Massage. 5. X. Entlassen: Gelenkkonturen normal; volle Funktion.

6. F. Sb., Arbeiter, 26 J. alt. Früher stets gesund. Ende September Ausfluß. Anfangs Oktober starke Schwellung des linken Handgelenks. Behandlung mit essigsaurer Tonerde, Umschläge und Schienenverbände. 
10. X. 1907 Poliklinik. Status: Das linke Handgelenk ist stark gerötet und geschwollen, ebenso Hand und Unterarm.

MaBe: Links 20, 21, 25 cm; rechts $17,17,23 \mathrm{~cm}$.

Hand steht in Flexions- und Abduktionsstellung. Die Strecksehnen des Daumens sind besonders stark befallen. Aktiv Spur von Beweglichkeit der Finger bei sehr starker Schmerzhaftigkeit. Phlegmonöse Form. 10. X. 1907. 22 stündige Stauung. Schmerzen lassen nach. 12.X. 1907. Passive Bewegungsmöglichkeit in ziemlicher Ausdehnung ohne Schmerzen. 13. X. Ganz auffallend rasche Besserung. Schnelles Abklingen der Entzündungserscheinungen. Ausgiebige aktive und passive Bewegungsmöglichkeit. 17. X. Nach 8 maliger 22 stündiger Stauung vollkommene Funktion des Gelenkes, nur noch geringe Schmerzempfindung bei Streckversuchen des Daumens. Massage. 21. X. Entlassen: Normale Gelenkkonturen und normale Funktion von Hand und Finger.

7. E. E., 26 J. alt, Melkefrau. Früher gesund. Seit 1 Jahr eitrigen AusfluB. Am 30. XII. plötzlich erkrankt unter Schüttelfrost und Fieber. Anschwellung und Rötung des rechten Handgelenks. Zunehmende Unbeweglichkeit von Hand und Finger. Poliklinik 3. I. 1908.

Status: Das rechte Handgelenk und seine Umgebung ist sehr stark geschwollen. MaBe: Rechts $20,20,23 \mathrm{~cm}$ gegenüber $16,16,20 \mathrm{~cm}$ links. Die Hand ist stark entzündlich gerötet, gespannt, ödematös. Unterarm, Hand und Finger zeigen ebenfalls Schwellung und Rötung. Die Hand steht in extremster Beugestellung. Bewegungen von Hand und Finger aktiv nicht möglich, passiv üuBerst schmerzhaft.

Stauung 22 stünd. ab 3. I. 1908 wird gut ertragen, wirkt rasch schmerzlindernd. Nachts Schlaf. 5. I. 1908. Passive Bewegungen schmerzlos in größerer Ausdehnung; aktive Fingerbewegungen. 10. I. 1908. Beugung und Streckung der Hand aktiv innerhalb $40^{\circ}$. 14. I. 1908. In der Staupause, Maße: 17, $17,20 \mathrm{~cm}$, nur noch mäBige Rötung, ganz schmerzfrei. 20. I. 1908. Rasche Wiederkehr der vollen Funktion. 21. I. 1908 bis 31. I. 1908. Bleibt wegen profuser Menses aus der Behandlung fort. 31. I. 1908. Rechtes Handgelenk ca. $1 / 2 \mathrm{~cm}$ verdickt. Funktion unbeschränkt.

8. Frl. H. Kl., 16 J. alt. Sonst gesund. Seit 8 Wochen eitrigen AusfluB. In der Nacht vom 13.14. V. 1905 starke Schmerzen im rechten Ellbogengelenk, keine Bewegungsfahigkeit; zumehmende Schwellung und Rötung besonders am Condyl, ext. Fieber. Poliklinik 17.V.1905. Fixierender Verband.

19. V. Status: Rechtes Ellbogengelenk ist sehr stark geschwollen, gerötet, ödematös; über dem Olekranon scheinbar Fluktuation.

Maße: etwas oberhalb der Epicondylen rechts $30 \mathrm{~cm}$, links 21,5 cm, über Olekranon und Gelenkfurche " 34 " 25 " $1 \mathrm{~cm}$ unterhalb davon $" 31,5 "$ " $" 23,5 "$

Schwellung ist am stärksten an der AuBenseite und Rückseite des Gelenks; völlige Bewegungsbeschränkung und auBerordentlich starke Druckempfindlichkeit und Schmerzhaftigkeit bei Bewegungsversuchen. Phlegmonöse Form.

Täglich Stauung 7 Stunden. Vorläufig fixierende Verbände (Dr. Guleke). 21. V. Schon ziemlich ausgiebige Flexions- und Extensions-, 
sowie Pro- und Supinationsbewegungen passiv ausführbar. 23. V. 12 Stunden Stauung. Starkes Ödem, keine Schmerzen mehr. Rötung ganz bedeutend zurückgegangen. Schienen fortgelassen. 24. V. Stauung von fruh 8 bis 12 Uhr nachts; darnach Hochlagerung. 25. V. Maße: 27,5, 29,5, $30 \mathrm{~cm}$. Bewegungen passiv schmerzfrei von $105^{\circ}-60^{\circ}$ ausführbar. Pro- und Supination normal. 5. VI. MaBe: 24,5, 27,5, 26,5 cm. Schmerzen nur noch bei ausgiebigen Bewegungen. Aktiv Extension bis $165^{\circ}$. Beugung bis $75^{\circ}$. Täglich 10 Stunden Stauung seit 1. VI. 10. VI. Nach 21 tägiger Stauung Konturen des Gelenks normal, keine Bewegungshinderung mehr, keine Schmerzen außer bei äußerster Extension; allmählich kurzere Stauung. 29. VI. Völlig normales Gelenk, nur nach längerem Hängenlassen des Armes noch leichte Schmerzen.

Nachuntersuchung Juli 1907: Völlig normales Gelenk ohne jede Schmerzempfindung mit voller Funktion.

9. B. M., Dienstmädchen, 18 J. alt. Früher stets gesund. AusfluB. Am 5. VIII. 1907 plötzlich sehr starke Schmerzen im rechten Ellbogengelenk, Schwellung und Rötung, Unbeweglichkeit. Aufnahme in ein Krankenhaus am 6. VIII. 1907. Nach Bericht des behandelnden Arztes handelte es sich um eine phlegmonöse Form der gon. Arthr. mit Fiebersteigerung bis $39^{\circ}$. Vom 6. VIII.-28. VIII. staute der Arzt tägl. 4-5 Stunden, länger wurde die Binde angeblich nicht ertragen. Es trat Besserung ein, Schwellung und Rötung traten allmählich zurück. Am 28. VIII. Poliklinik.

Status: Rechtes Ellbogengelenk sehr stark geschwollen, gerötet, ödematös. Die Schwellung erstreckt sich auf Oberarm und Unterarm, besonders auf der Streckseite des Oberarmes starke Schwellung. Druck auf die Bicepssehne sehr schmerzhaft. Bewegungen im Ellbogengelenk gehemmt. Beugung $90^{\circ}$, Streckung bis $120^{\circ}$, äuBerst schmerzhaft.

Vom 29. VIII.-6. IX. tägl. 22 stündige Stauung. Die entzündlichen Erscheinungen treten sehr rasch zurtick. Die Bewegungen ausgiebiger und ganz schmerzfrei. 8. IX. Bewegungen im Gelenk vollständig frei, mäBige Schwellung, Staubinde fort, Massage. Am 13. IX. entlassen: Gelenkkonturen normal, Beweglichkeit wie links. Beiderseits infolge rachitischer Veränderungen geringe Extensionsbeschränkung $\left(10^{\circ}\right)$.

10. Sch., 20 J. alt, Nähterin. Seit Oktober 1907 in ärztlicher Behandlung wegen Trippers. Am 9. XI. 1907 plötzlich beim Maschinennähen heftige Schmerzen im linken Ellbogengelenk, das in den folgenden Tagen sehr stark anschwillt. Arzt verordnet kalte Umschläge und Pulver. Am 14. XI. Poliklinik.

Status: Das linke Ellbogengelenk ist mächtig geschwollen, entzündlich gerötet und ödematös. Die Konturen des Gelenks sind ganz verwischt.

Maße: Links 32, 33,5, $32 \mathrm{~cm}$, rechts 20, 21, $22 \mathrm{~cm}$. Die Schwellung und Rötung setzt sich nach oben bis zur Schulter fort, nach unten bis auf die Finger. Im Sulcus bicipitalis internus zieht sich vom Epicondylus medialis bis etwa zur Mitte des Oberarms eine ca. 2 Finger breite brettharte Induration, die besonders stark druckempfindlich ist. Das Gelenk steht in Mittellage fest fixiert; jede Bewegung aktiv und passiv unmöglich. ÄuBerste Schmerzhaftigkeit; Hand- und Fingerbewegungen rufen Schmerzäußerungen hervor. Schwere phlegmonöse Form. 
14./15. XI. 22 stündige Stauung. Pat. schläft die erste Nacht wieder, die Schmerzen sind nur ganz geringe. 16. XI. Passive Bewegungen in mäßigen Grenzen möglich. Stauung bis 22. XI. Spontan schmerzfrei; aktive Bewegungen noch unmöglich; passive Bewegungen im $\triangle$ von $30^{\circ}$ ausführbar.

Pat. bleibt vom 22. XI.-3. XII. wegen Angina aus der Behandlung fort. Angeblich läßt sie sich die Binde täglich mehrere Stunden anlegen, um die Schmerzen fortzunehmen.

Am 3. XII. Maße: Links 28, 29, $29 \mathrm{~cm}$, rechts 20, 21, $21 \mathrm{~cm}$, heftige Entzündungserscheinungen noch vorhanden. Passiv Spur von Bewegung möglich. Ab 3. XII. wieder 22 stündige Stauung, täglich zunehmende Besserung. 7. XII. Geringe aktive Beweglichkeit. Schwellung und Rötung geringer, Haut runzelt sich. 11. XII. Das Stauungsödem wird nicht mehr so feurig. Die Induration im Sulcus bicipitalis internus wird weicher und kleiner. 13. XII. Exkursionswinkel ca. $80^{\circ}$. Pro- und Supination nur noch wenig beschränkt. 21. XII. Entlassen: Schmerzfrei. Normale Gelenkkonturen, unbeschränkte Funktion.

11. R., Frau, 45 Jahre alt. Früher gesund. Schon längere Zeit eitrigen AusfluB. Am 7. X. in der Nacht erwacht Pat. an heftigen Schmerzen im rechten Schultergelenk. Am 8. X. besteht eine starke Schwellung und Rötung der rechten Schultergegend; starke Schmerzhaftigkeit; Unbeweglichkeit des Armes, seitdem ohne Schlaf. Am 10. X, 1907 Poliklinik.

Status: Die ganze rechte Schulterwölbung ist ungemein und in rundlicher Gestaltung vermehrt. Akromion und Processus coracoideus sind verwischt. Die Weichteile sind stark entzündlich gerötet und ödematös geschwollen. Der rechte Arm hängt am Thorax herab; alle Bewegungen im Schultergelenk sind aktiv unmöglich. Der Humeruskopf ist völlig fixiert; passiv ist eine Spur Elevation in der Sagittal- und Frontalebene und eine Spur Rotation, doch nur unter Mitbewegung der Scapula möglich. Bloße Berührung äußerst schmerzhaft.

10. X. 22 stündige Stauung. Pat. schläft zum erstenmal wieder die ganze Nacht. Schmerzen fort. Der Schulterschlauch ist namentlich bei Bettruhe etwas unbequem. 12. X. Rapide Besserung. Elevation und Abduktion ohne Scapulamitgehen ca. $45^{0}$; Rotation ziemlich ausgiebig; Schmerzen fort. 12. X.-16. X. Täglich Stauung unter fortschreitender Besserung bis zur Norm.

Nach 8 tägiger Stauung - 10. X.-18. X. - geheilt entlassen. Normale Schulterwölbung, entzündliche Erscheinungen ganz gehoben; schmerzfrei; vollkommene Funktionstüchtigkeit des rechten Armes.

12. H. Kr., Dienstmädchen, 28 Jahre alt. Früher nie ernstlich krank. Seit einigen Wochen eitriger AusfluB. Am 23. XII. starke Zerrung des linken Armes. Am 31. XII. plötzlich heftige Schmerzen im linken Schultergebiet, Anschwellung und Unbeweglichkeit. Am 4. I. Poliklinik.

Status: MäBige Schwellung der linken Schultergegend, Haut darüber gerötet und heiß. Kein nachweisbarer Erguß im Gelenk. Der Humeruskopf ist fest fixiert. Aktive Bewegungen nicht möglich, passiv Abduktion 
und Elevation nach vorn ca. $\mathbf{1 5}^{0}$, aber nur unter Mitbewegung der Scapula. Sehr große Schmerzhaftigkeit.

4. I. 1908. Stauung. Wird sofort sehr schmerzlindernd empfunden. 8. I. 1908. Passive Rotationen des Kopfes in mäBigen Grenzen ausführbar; aktiv: geringe Elevationen nach vorn und nach der Seite. 16. I. 1908. Erheben des Armes vorn rechts seitlich bis zur Horizontalen ohne Scapulamitgehen. Über dem Akromion geringe Hautabschürfungen. 28. I. 1908. Humeruskopf in voller Ausdehnung d. h. wie rechts rotierbar. 30. I. 1908. Vollkommen freie Beweglichkeit des Armes nach allen Richtungen hin. Geheilt entlassen.

13. W. W., 35 Jahre alt, Kaufmann. Sonst gesund. Seit 31. I. Tripper. Am 5. II. Conjunctivitis gon. und zu gleicher Zeit heftige Schmerzen und zunehmende Unbeweglichkeit der linken Schulter. 8. II. Poliklinik.

Status: Schwellung der linken Schultergegend. Haut gerötet und heiB. Aktive Bewegung wegen starker Schmerzhaftigkeit behindert. Passiv geringe Rotation, Elevation nach vorn und zur Seite ca. $30^{\circ}$.

8. II. Stauung. Stauung wird als lästiger Druck empfunden. Ödem ganz enorm. Oberarmumfang $6 \mathrm{~cm}$ starker wie rechts. 16. II. 1908. Wirkung der Stauung setzt sehr rapide ein. Volle Bewegung des Arms. 17. II. 1908.-22. 2. 1908 Massage. Geheilt entlassen.

14. E. M., Frau, 39 Jahre alt. Sonst gesund. Vor $21 / 2$ Wochen Zerrung des linken Handgelenks, nachts heftige Schmerzen, tags darauf starke Schwellung und Unbeweglichkeit des linken Handgelenks, Fieber. Trotz Schienenverbände keine Besserung, stets so starke Schmerzen, daß Pat. in den $2 \frac{1}{2}$ Wochen nicht hat schlafen können. Im Nov. Abort. 16. III. Gelber Ausfluß. 7. VI. 1905 Poliklinik.

Status: Linke Handgelenksgegend, besonders am Dorsum, sehr stark geschwollen, ödematös, livide verfärbt, äuBerst druckempfindlich. Maße: $18,5,19,22,5 \mathrm{~cm}$ gegenüber $16,16,5,19 \mathrm{~cm}$ rechts. Das Ödem erstreckt sich auf die Finger. Hand und Finger unbeweglich. Phlegmonöse Form.

Täglich 8 Stunden Stauung; es gelingt sehr leicht, kräftiges Ödem zu erzeugen. Schon vom ersten Tage an viel weniger Schmerzen. Nachts Schlaf. Suspension.

Am 2. VI. Fingerbewegungen schon innerhalb $25^{0}$, Beugung im Handgelenk ca. $10^{\circ}$ aktiv ausführbar. Es besteht ziemlich beträchtliches Ödem, zeitweise Stechen im Kleinfingerballen, Pat. fühlt sich leidlich wohl, täglich 12 Stunden Stauung. 1. VII. Die Finger können bis etwa $60^{\circ}$ gebeugt werden, leichte Flexions- und Extensionsbewegungen im Handgelenk möglich, bei forciertem Versuch noch Schmerzen. 26. VII. Handgelenk noch leicht verdickt. Streckung bis $40^{\circ}$. Beugung bis $25^{\circ}$ ausführbar, Ab- und Adduktion ziemlich ausgiebig. Pro- und Supination normal. Finger können bis zur Hälfte des Normalen gebeugt werden, der kleine Finger bleibt dabei wesentlich zurück.

Nachuntersuchung 6. VI. 1907. Stauung wurde nach Angabe der Pat. bis Mitte August fortgesetzt. Pat. ist beschwerdefrei. Handgelenk vollkommen frei beweglich. Beim Schließen zur Faust bleibt Dig. II und $\mathrm{V}$ ca. $4-5 \mathrm{~cm}$ vom Handteller entfernt. 
15. F. W., Frau, 23 Jahre alt. Früher gesund. Am 13. VII. 1906 partus. Am 17. VII. Schüttelfrost, Fieber, starke Schmerzen im linken Handgelenk. Kind Conjunctivitis gon. Behandlung mit fixierenden Verbänden. Am 1. III. Poliklinik.

Status: Schwere phlegmonöse Arthritis gon. manus sin. mit völliger Unbeweglichkeit von Hand und Finger (Dr. Volkmar).

Nach 5 wöchiger täglich 12 stündiger Stauung mit guter Funktion der Hand entlassen.

Nachuntersuchung am 16. X. 1907. Pat. hat zeitweise Schmerzen im linken Handgelenk. Die motorische Kraft von Hand und Arm ist vermindert. Gelenkkonturen normal; Maße beiderseits: 16, 16, $20 \mathrm{~cm}$. Geringe Krepitation im Radiocarpalgelenk. Bewegungen im Handgelenk wie rechts; nur geringe Streckbehinderung $\left(5^{0}\right)$. Finger vollkommen intakt.

16. Gr. M., 18 Jahre alt, Dienstmädchen. Früher gesund. Am 11.X. 1906 plötzlich erkrankt unter heftigen Schmerzen im linken Handgelenk; tags darauf Schwellung, Rötung, Unbeweglichkeit von Hand und Finger. Behandlung mit Einreibungen und Packungen. Am 1. XI. Poliklinik.

Status: Starke Schwellung und Rötung des linken Handgelenks. Die periartikulären Weichteile, besonders die Strecksehnen, stark befallen; Fingergelenke dick und gerötet; Bewegungen der in Streckstellung fixierten Hand und Finger aktiv unmöglich, passiv heftige Schmerzen.

Ab 1. XI. Stauung 12 Stunden. Schmerzen sofort geringer. Nachts Schlaf. Am 5. XII. wesentliche Besserung. Flexion ca. $50^{\circ}$, Finger können ganz in die Hand eingeschlagen werden. 21. XII. Beugung fast normal; Pro- und Supination frei. Extension noch behindert.

Nachuntersuchung 19. IX. 1907. Angeblich noch bis Ende Jan. 1907 gestaut. Hat Schmerzen im Gelenk bei Witterungswechsel. Gelenkkonturen normal. Rauhe Krepitation. Bewegungen der Hand bis auf geringe Differenz $\left(5-10^{\circ}\right)$ in Beugung und Streckung wie rechts. Finger frei.

17. B. G., Kutscher, 19 Jahre alt. Früher nie ernstlich krank, Dezember 1905 Ulcus molle und Tripper. Anfang Dezember 1906 plötzlich auftretende Schmerzen im Knie und linken Handgelenk. Später im rechten Handgelenk. Poliklinik 28. XII. 1906.

Phlegmonöse Arthritis gon. des rechten Handgelenks. Finger and Hand ganz unbeweglich (Dr. Müller). Stauung 8 Wochen täglich 12 Stunden; danacil mit voller Funktion entlassen.

Nachuntersuchung am 13. IX. 1907. Handgelenk schmerzfrei. Motorische Schwäche der Hand. Oberarmmuskulatur etwas atrophisch. Gelenkkonturen normal. Funktion der Hand und Finger wie links.

18. A. H., 18 Jahre alt, Blumenbinderin. Früher stets gesund. Seit Mitte April eitrigen AusfluB. Am 25. IV. blitzartigen Schmerzanfall über dem Metacarpus V; Anschwellung der Beugesehne des fünften Fingers rechts, in der Nacht schwillt das rechte Handgelenk an. Arzt legt Gipsverband an, während dieser Zeit entsetzliche Schmerzen. Nach 8 Tagen Entfernung des Gipsverbandes. Heiße Umschläge alle 5 Minuten lang, Tag und Nacht 3 Wochen lang; kein Schlaf, daneben durch reichliche Salizyl-Dosen Übelkeit, Erbrechen, hohes Fieber. Am 15. V. 1907 nach 3 Wochen - Poliklinik. 
Zur Beh. d. Arthr. gonorrh. d. großen Gelenke mittels Stauungshyperämie. 69

Status: Rechtes Handgelenk und seine Umgebung, bis herauf zum Ellbogen und peripher auf die Finger übergreifend, ist diffus polsterartig geschwollen. Die Haut ist stark entzundlich gerötet und gespannt, odematös. Maße: 21, 22, $22 \mathrm{~cm}$ rechts, 15, 15, $16 \mathrm{~cm}$ links. Hand and Finger stehen vollstanelig fixiert in Streckstellung. Alle Bewegungen aktiv und passiv sind vollständig aufgehoben. Es besteht spontan eine sehr starke Schmerzhaftigkeit, Bewegungen veranlassen den Patienten zum Aufschreien. Sehr schwere phlegmonöse Form mit starker Beteiligung der Beugesehnen.

Am 16. V. Stauung. Binde liegt bis 2 Uhr nachts. Schmerzen haben sofort nachgelassen. 17. V. Binde von 6-9 Uhr morgens. Pat. schlaft die ganze Nacht. 20. V. Schwellung schon zurückgegangen. Maße: $20 \mathrm{~cm}$, 21,5, $21 \mathrm{~cm}$. Spontan schmerzfrei. Nur bei passiven forcierten Bewegungen noch Schmerzen. 30. V. 1907. Die Entzündungserscheinungen sind fast ganz geschwunden. Die Bewegungsfähigkeit von Hand und Finger setzt äuBerst langsam ein. Auch ein 8tägiger Versuch - vom 1. VII.-9. VII. - im Handsaugglas bringt keine Fortschritte. Deshalb wieder Stauung ab 10. VII. 15. VII. Beugung $160^{\circ}$, Streckung $170^{\circ}$. Pro- und Supination bis zur Hälfte des Normalen. Adduktion ganz behindert. Finger bis $2 \mathrm{~cm}$ von der Hohlhand, kleiner Finger ganz unbeweglich. 20. VII. Beugung $150^{\circ}$, Streckung $160^{\circ}$, Pro- und Supination frei. $A b-$ und Adduktion bis zur Hälfte des Normalen. Finger können mit Ausnahme des fünften zur Faust eingeschlagen werden. Vom 24. VII. ab nur noch Massage und heiße Handbäder. Entlassen am 13. VIII. nach 13 Wochen.

Befund: Die motorische Kraft der Hand und des rechten Armes ist herabgesetzt. Über der Vola manus, besonders uber dem Metacarpus V besteht noch derbe Schwellung. Im Radiocarpalgelenk geringe Krepitation. Handgelenk etwas verdickt. Maße: 16, 16, $17 \mathrm{~cm}$. Funktion beschränkt: Beugung $150^{\circ}$, Streckung $150^{\circ}$ d. h. Exkursionswinkel $60^{\circ}$ (gegenüber $120^{\circ}$ links). Ab- und Adduktion bis zur Halfte behindert. Pro- und Supination frei. Finger können vollständig in die Hand eingeschlagen werden; nur der fünfte bleibt bis auf $5 \mathrm{~cm}$ von der Hohlhand entfernt.

19. P. F., 23 Jahre alt, Dreher. Früher nie ernstlich krank. Im Februar geschlechtskrank. Am 13. V. nachmittags $6 \mathrm{Uhr}$ beim Spaziergang plötzlich sehr heftige Schmerzen im rechten Handgelenk. Auf der Unfallstation Schienenverband. In der Nacht Steigerung der Schmerzen; starke Anschwellung des rechten Handgelenks und der Finger.

14. V. Hand und Finger völlig unbeweglich, ab 15. V. ärztliche Behandlung; $2^{1 / 2}$ Wochen Schienenverband, dann $2^{1 / 2}$ Wochen Gipsverband, dann Mitella; da keine Besserung eintritt, kommt Pat. am 1. VII. 1907 7 Wochen nach dem Eintritt der Erkrankung - in die Poliklinik.

Status: Das rechte Handgelenk ist etwas geschwollen, Maße: rechts $17,18,21 \mathrm{~cm}$, links $16,17,20 \mathrm{~cm}$, es besteht eine mäBige Rötung der Haut und geringes Ödem des Unterarmes. Die Hand hängt in Volarflexion und ist radialwärts verschoben, sie ist vollig fixiert; jede aktive und passive Bewegung ist unmöglich, die Finger sind in allen Gelenken gestreckt; sehr lebhafte Schmerzhaftigkeit.

Vom 5. VII. ab täglich Stauung bis nachts 11 Uhr, sehr heißes 
Ödem, Schmerzen sofort gelindert, Stauung gut ertragen. In der 1. Woche außer der Schmerzlinderung keine Ȧnderung. 6. VII. ab 22 stündige Stauung, darnach 2 Stunden Suspension. 15. VII. Die Schwellung ist zurückgegangen, die Rötung hat einer lividen Färbung Platz gemacht; Finger können ziemlich ausgiebig bewegt werden. Handgelenk passiv innerhalb $\measuredangle 30-40^{\circ}$ schmerzfrei beweglich. 25. VII. Aktive Bewegung der Hand setzt langsam ein. Beugung und Streckung ca. 80\%. Pro- und Supination fast normal. Adduktion noch behindert. 1. VIIT. Beugung und Streckung innerhalb $60^{\circ}$. Pro- und Supination normal. 10. VIII. Beugung and Streckung nur noch wenig behindert. Am 12. VIII. Stauung fortgelassen, nur noch Massage bis zum 18. VIII. Am 19. VIII. - nach 7 Wochen - arbeitsfahig entlassen.

SchluBbefund: Das rechte Handgelenk ist $1 / 2 \mathrm{~cm}$ dicker wie das linke. Motorische Kraft des rechten Armes etwas herabgesetzt. Schmerzfrei, Beugung und Streckung ist gegenüber links ganz wenig (ca. 10 ${ }^{\circ}$ ) behindert. Alle übrigen Bewegungen, auch der Finger vollkommen frei.

20. B., Fran, 27 Jahre alt. Früher gesund, seit der Verheiratung AusfluB. Vor $5 \frac{1}{4}$ Monaten plötzlich Schmerzen im linken Handgelenk, Fieber; starke Rötung und Schwellung; Unbeweglichkeit von Hand und Fingern. Behandlung mit Moorbädern, Fango, Handbädern, Schienenverbänden. Da keine Besserung eintritt am 9. IX. 1907 - nach 21 Wochen - Poliklinik.

9. IX. 1907 Status: Geringe Schwellung des linken Handgelenks, kein ErguB; fühlt sich heiB an, bei Bewegungsversuchen starke Schmerzen und Krepitation. Aktiv: Beugung und Streckung innerhalb eines $\Varangle$ von $20^{\circ}$. Ab- und Adduktion ganz behindert. Pro- und Supination frei, beim FaustschlieBen bleiben die Finger $3 \mathrm{~cm}$ vom Handteller entfernt. 9. IX. bis 20. IX. Tảglich 22 stündige Stauung; die gut etragen wird. Schmerzen lassen nach, Bewegungen werden ausgiebiger. Beugung und Streckung innerhalb $50^{\circ}$. Abduktion bis zur Hälfte des Normalen. Finger bis ${ }_{12} \mathrm{~cm}$ vom Handteller. Stauung bis 1. X. 1907. 1. X.-8: X. Massage.

Entlassungsstatus: Pat. ist zurzeit ganz schmerzfrei. Beugung und Streckung innerhalb $60-70^{\circ}$, Abduktion bis zur Hälfte des Normalen, Adduktion behindert; Finger werden ganz zur Faust eingeschlagen.

21. H. Sch., 19 Jahre alt. Früher gesund. 1906 eitrigen Ausfluß. Seit Mitte August 1907 wieder starken Ausfluß. Am 1. IX. 1907 plötzlich sehr starke Schmerzen im rechten Handgelenk; Fieber und schlechtes Allgemeinbefinden. Schwellung und Rötung der Hand, Schmerzen besonders bei Bewegungen. Arzt verordnet Bäder, Einreibungen, doch ohne Erfolg. 12. IX. Poliklinik.

Status: ÄuBerlich keine Gelenkveränderungen; das Handgelenk fühlt sich heiß an; geringgradige ulnare Abduktion der Hand. Bewegungen im Handgelent auBer der Abduktion fast normal, doch enorm schmerzhaft. Beim Versuch, eine Faust zu machen, bleibt der dritte und vierte Finger wesentlich zurück (Arthralgie).

12. IX, Aufnahme in die Klinik. Die Stauung nimmt sofort den Schmerz und ermöglicht schon nach einigen Stunden alle Bewegungen der Hand und Finger. 13. IX. Ohne Binde noch Schmerzempfindung. Stauung 
Zur Beh. d. Arthr. gonorrh. d. großen Gelenke mittels Stauungshyperämie. 71

bis 16. IX. 1907 täglich 22 Stunden. 17. IX. 1907 entlassen. Schmerzfrei. Gelenkfunktion normal.

22. K. M., 18 Jahre alt, Dienstmädchen. Früher gesund. Am 10. VII. ohne äußere Ursache Schmerzen im rechten Handgelenk, später sehr starke Anschwellung und Rötung der rechten Hand und des rechten Unterarmes, Fieber, schlechtes Allgemeinbefinden. Behandelt sich selbst mit Einreibungen, Einpackungen und hält Bettruhe. Da die Hand immer mehr versteift, kommt Pat. nach 9 Wochen in die Poliklinik.

Status: 12. IX. Rechtes Handgelenk äußerlich kaum verändert. Haut darüber livide verfärbt, Gelenkgegend fühlt sich heiß an. Bewegung von Hand und Finger beschränkt, Beugung und Streckung innerhalb $\measuredangle 10^{\circ}$. Pro- und Supination zur Hälfte behindert. Ab-und Adduktion, sowie Rotation unmöglich. Finger bleiben beim Faustmachen $7 \mathrm{~cm}$ von der Hohlhand entfernt. Bei passiven Bewegungsversuchen starke Krepitation im Radiocarpalgelenk und lebhafte Schmerzen.

12. IX. Staung. Heiße Stauung erst nach einem heißen Handbad. 16. IX. Beugung und Streckung innerhalb $\Varangle$ von $30^{\circ}$. Stauung bis 1. X. 1907. Entlassen 1. X. Pat. ist beschwerdefrei. Bengung und Streckung innerhalb $70^{\circ}$. Abduktion normal. Adduktion etwas behindert. Pro- und Supination frei. Keine Krepitation. Finger frei beweglich.

23. M. E., 18 Jahre alt, Dienstmädchen. Früher gesund. Ende Juli starke Schwellung des rechten Handgelenks; zunehmende Schmerzhaftigkeit und Unbeweglichkeit von Hand und Finger. Fluor. Mitte August Aufnahme in ein Krankenhaus. In der ersten Woche fixierender Verband; sodann Stauung täglich 2 Stunden. Nach 3 Wochen auf Wunsch aus dem Krankenhaus entlassen. Doch bestand noch die Schwellung und Unbeweglichkeit der Hand weiter. Seit 1. X. wieder stärkere Schmerzen. Poliklinik am 14. X. 1907.

Status: Mäßige Schwellung des rechten Handgelenks. Maße: 17,5, $18,5,17 \mathrm{~cm}$ rechts, links $16,17,15 \mathrm{~cm}$. Blaurote Verfärbung der Haut, fühlt sich heiB an, Druck auf das Handgelenk äuBerst schmerzhaft. Bewegungen sehr beschränkt, aktiv nur Volarflexion von ca. $10^{0}$ möglich, Finger frei.

Stauung vom 15. X,-20. X. Auf Wunsch entlassen. Handgelenk bei schwerer Arbeit noch etwas schmerzhaft. Nur noch geringe Extensionsbehinderung $\left(10^{\circ}\right)$, sonst alle Bewegungen frei.

24. S. A., 24 Jahre alt, Fräulein. Sonst gesund; seit lảngerer Zeit AusfluB. Am 10. X. plötzlich erkrankt mit Schwellung und Rötung des linken Handgelenks. Zunehmende Unbeweglichkeit von Hand und Finger. Behandlung mit Schienenverbänden. Am 26. X. 1907 Poliklinik.

Status: Linkes Handgelenk stark geschwollen, deutlicher Erguß im Gelenk, Hand steht in Flexionsstellung, ulnarwarts verschoben. Maße: $18,18,5,18 \mathrm{~cm}$ gegen $16,5,16,16 \mathrm{~cm}$. Die einzelnen Fingergelenke sind dick, lebhaft gerötet. Bewegungen von Handgelenk und Finger aktiv unmöglich, passiv Beugung der Hand ca. $30^{\circ}$. Pro- und Supination bis zur Hälfte des Normalen. Spontan starke Schmerzhaftigkeit.

Ab 26. X. Stauung täglich 22 Stunden, 2 Stunden Suspension. I. XI. Die Entzündungserscheinungen sind fast vollständig gewichen; noch Spur 
von Gelenkergu $B$ vorhanden, Bewegungen sind ansgiebig und schmerzfrei. 5. XI. Bewegungen in normaler Ausdehnung möglich. Massage. 8. XI. Entlassen. Ohne Beschwerden. Normale Funktion von Hand und Finger.

25. Franz S., Arbeiter, 25 J. alt. Früher gesund. Im August 1907 einige Tage AusfluB. Am 11. Sept. sehr starke Schwellung des rechten Handgelenks, zunehmende Unbeweglichkeit. Arzt verordnet Salizyl und Einreibungen. Da ohne Erfolg, am 14. X. 1907 - nach ca. 5 Wochen - Poliklinik.

Status: Rechtes Handgelenk und seine Umgebung sind sehr stark geschwollen. Maße: 24, 24, 28 gegen 17, 17, $21 \mathrm{~cm}$ links. Die Haut ist stark gespannt, glänzend, heiß gerötet und ödematös. Handrücken polsterartig, Handteller ganz verstrichen, Unterarm ödematös. Die einzelnen Finger sind wurstförmig verdickt, die Interphalangealgelenke mit prall gespannter Kapsel äußerst druckschmerzhaft. Über den Beugesehnen in der volaren Handgelenksfurche lebhafte Rötung und starke Druckempfindlichkeit.

Die Hand hängt herab in Flexionsstellung, ist radialwärts verschoben und subluxiert. Bewegungen von Hand und Finger aktiv und passiv unmöglich. Enorme Schmerzhaftigkeit.

14. X. 190722 stündige Stauung. Lebhaft feuriges Stauungsödem. Schmerzen lassen sofort nach. Abends $8 \mathrm{Uhr}$ bei der Kontrolle aktiv leichte Fingerbewegungen; passiv Spur von Handbewegungen. 18. X. 1907. Spur von aktiver Handbewegung. Finger besser. Wegen des mächtigen Ödems 24 Stunden Stauung fort. Suspension. 24. X. Entzündungserscheinungen geringer. MaBe: $21,21,25 \mathrm{~cm}$. Aktiv Beugung und Streckung ca. $40^{\circ}$. Pro- und Supination bis zur Hälfte. $A b-$ und Adduktion noch beschränkt und schmerzhaft. Pathologische Handstellung durch Lagerung in der Mitella zu korrigieren versucht. 28. X. In der Staupause nur noch mäBige Schwellung. 19, 19, $23 \mathrm{~cm}$. Haut runzelt sich. Handstellung gebessert. 2. XI. Entzündliche Symptome gehoben. Schmerzfrei. Beugung und Streckung ca. $70^{\circ}$. $\mathrm{Ab}$ - und Adduktion bis zur Hälfte. Pro- und Supination frei; Finger bis zu $1 \mathrm{~cm}$ vom Handteller entfernt. 3. XI. Staung fort. Massage bis Anfang Dezember.

Anfang Dezember entlassen. In der Ruhe und in der Arbeit schmerzfrei. Motorische Kraft der Hand noch stark herabgesetzt. Handgelenk dorsal etwas verdickt. 17, 18, $21 \mathrm{~cm}$. Hand eine geringe Spur radialwärts verschoben. Beugung und Streckung $80^{\circ}$ gegen $120^{\circ}$ links. Adund Abduktion bis zur Hälfte. Pro- und Supination frei. Finger vollkommen frei beweglich.

26. W. B., 20 J. alt, Handlungsgehilfe. Früher gesand. Vor $1 / 4$ Jahr Tripper. Mitte Februar 1905 sehr schmerzhafte starke Anschwellung des rechten Ellbogengelenks, Bewegungsbeschränkung, Fieber. Trotz feuchter Umschläge, Einreibungen und fixierender Verbande keine Besserung. Poliklinik 18. III. 1905.

Status: Hochgradige Anschwellung des rechten Ellbogengelenks, ödematöse Anschwellung der Weichteile, Rötung. Maße: 28, 31, 30 cm gegen $21,25,22,5 \mathrm{~cm}$. Völlige Fixation, starke Schmerzhaftigkeit bei Druck und Bewegungsversuchen. Die Schwellung erstreckt sich bis zur Mitte des Vorderarms, nach oben bis Handbreite über dem Ellbogengelenk. Muskulatur atrophisch. Phlegmonöse Form. 
Zur Beh. d. Arthr. gonorrh. d. großen Gelenke mittels Stauungshyperämie. 73

Stauung tägl. 1 Stunde, schon nach 2 Tagen wesentliche Besserung; Nachlassen der Schmerzen und der Druckempfindlichkeit.

30. III. 1905. Schwellung bedeutend zurückgegangen. $24,28,27,5 \mathrm{~cm}$. Im Gelenk passive Bewegungen von $1 \mathrm{R}-75^{0}$ schmerzfrei. Hand völlig beweglich bis auf Extension. 17. IV. 1905. Es besteht nur noch ganz geringe Anschwellung in der Gegend des Olekranon, hier auch noch Druckempfindlichkeit. Maße: 22, 26, 24,5 cm. Pat. schläft seit 8 Tagen, in der Hand nur wenig Kraft. Tägl, bis 4 Stunden Stauung. 24. V. 1905. Aktive Bewegungen im Ellbogen innerhalb $60^{\circ}$, Supination von der Mittelstellung aus nur $45^{0}$ ausführbar. Keine Druckempfindlichkeit, keine Schmerzen mehr, nur bei forcierten Bewegungsversuchen.

Nachuntersuchung 6. VI. 1907. War angeblich nach dem 24. V. 1905 noch längere Zeit - 2-3 mal wöchentlich - in Staubehandlung. Pat. ist beschwerdefrei, ist tätig als Schreiber. Maße: Rechts 22, 25, $24 \mathrm{~cm}$ wie links. Motorische Kraft des Arms wesentlich herabgesetzt. Funktion beschränkt. Beugung bis $75^{\circ}$, Streckung bis $150^{\circ}$. Pronation bis über die Hälfte des Normalen, Supination etwas behindert.

27. E. W., 29 J. alt, Frau. Früher nie ernstlich krank. Seit 10. XI. $1906 \mathrm{ab}$ Gelenkschmerzen in allen größeren Gelenken, seit 16. XI. 1906 ab Lokalisation im rechten Ellbogengelenk. AusfluB. Ärztliche Behandlung: Innerlich Salizyl und lokale Umschläge mit essigsaurer Tonerde. Da keine Besserung am 3. XII. Poliklinik.

Status: Das rechte Ellbogengelenk ist sehr stark geschwollen. Die Haut darüber stark gerötet und ödematös; die Schwellung greift auf Olekranon und Unterarm über; besonders an der Streckseite des Oberarms starke Schwellung. Das Gelenk steht in beinaher rechtwinkliger Beugung und Pronation fest; Bewegungen aktiv und passiv nicht ausführbar; starke Schmerzhaftigkeit. Phlegmonöse Form.

Ab 4. XII. tägl. 6-8 Stunden Stauung; sie wird gut ertragen, die Schmerzen lassen bald nach. 15. XII. Schwellung etwas zurückgegangen; passive Bewegungen in geringem Grade ohne Schmerzen möglich; 10 bis 12 stündige Stauung täglich. 28. XII. Ganz erhebliche Besserung; aktive Bewegungen ausgiebiger. Beugung ca. $70^{\circ}$, Streckung ca. $140^{\circ}$. 15. I. 1907. Stauung bis heute täglich 12 Stunden fortgesetzt. Rötung und Schwellung verschwunden. Aktive Bewegungen fast normal. 15./31. I. 1907. Massage. 1. VIII. 1907. Nachuntersuchung: Pat. ist beschwerdefrei; das Ellbogengelenk zeigt normale Konturen; geringe leichte Krepitation, Bewegungen bis auf ganz geringe Streckbehinderung (ca. 5-10 $)$.

28. G. Kl., 26 J. alt, Expedientin. Früher stets gesund. Am 10. Juni plötzlich Schmerzen im linken Arm; tags darauf Anschwellung des rechten Ellbogengelenks, heftige Schmerzen. Arzt verordnet Linimente, Einpackungen doch ohne Erfolg. Poliklinik am 5. VII. 1907 (25 Tage nach der Erkrankung).

Status: Kolossale Schwellung des rechten Ellbogengelenks, Haut darüber gespannt, gerötet, glänzend. Schwellung und Rötung erstreckt sich bis zur Schulter und nach vorn bis zur Hand; typische spindelförmige Anschwellung. Maße: Rechts 30, 33, $32 \mathrm{~cm}$, links 22, 23, $22 \mathrm{~cm}$. Das Gelenk steht in stumpfwinkliger Beugung bei supiniertem Vorderarm. 
Aktive Bewegungen nach allen Richtungen hin unmöglich, passiv ebenso. Bloße Berührung bringt die Pat. zum Aufschreien. Finger können wegen schmerzhafter Spannung nicht zur Faust eingeschlagen werden. Fieber, Schlaflosigkeit; ganz schlechtes Allgemeinbefinden. Schwere phlegmonöse Form.

Ab 5. VII. 190722 stündige Stauung, die Schmerzhaftigkeit läßt nach, in der Nacht Schlaf. Am 10. VII. 1907 Schwellung geringer. Maße: 27, $30,28 \mathrm{~cm}$. Passive Bewegungen im mäßigen Grade ausführbar; unter der Binde keine Schmerzen. 20. VII. Schwellung fast vollständig geschwunden. Maße: 24, 25, $23 \mathrm{~cm}$. Aktiv Streckung bis $160^{\circ}$, Beugung $75^{\circ}$. Pro- und Supination bis zur Hälfte des Normalen. 25. VII. Entzündungserscheinungen gehoben, Runzelung der Haut, Gelenk fühlt sich noch heiß an. Streckung bis $170^{\circ}$, Beugung $50^{\circ}$. 1. VIII. Stauung fort, verläBt vorzeitig die Behandlung.

Nachuntersuchung 0kt. 1907: Gelenkkonturen normal. Aktive Bewegungen frei bis auf geringe Extensionsbehinderung (ca. 10 ${ }^{\circ}$ ).

29. M., Arbeiter, 21 J. alt. Früher nie ernstlich krank. Seit 18 Tagen Tripper. Seit 10 Tagen Schmerzen, Anschwellung und Unbeweglichkeit des rechten Ellbogengelenks. Am 2. VI. Aufnahme in die Klinik.

Status: Das rechte Ellbogengelenk ist stark geschwollen, die Haut darüber gerötet, heiß und ödematös. Das Gelenk steht in stumpfwinkliger Stellung fixiert, aktive und passive Bewegungen unmöglich, wegen starker Schmerzhaftigkeit. An der Innenseite des Oberarms im Sulcus bicipit. int. eine heiße derbe Infiltration, die sehr schmerzhaft ist.

Vom 2./12. VI. tägl. 18-22 stündige Stauung. Das Infiltrat wird in ein hartes derbes Gewebe ungewandelt. Ab 14. VI. Heißluftbehandlung. 20. VI. Geheilt entlassen. Normale Gelenkkonturen. Volle Funktion.

30. Wilh. F., Gerichtsdiener, 30 J. alt. Juni 1907 Tripper. Am 16. August nachts erwacht Pat. an Schmerzen im rechten Ellbogengelenk; tags darauf Schwellung und Rötung der Haut, hohes Fieber. Behandlung mit Umschlägen, Aspirin, später Bàder. Trotzdem versteift der Arm mehr und mehr, so daß Pat. am 26. X. 1907 - nach 10 Wochen - in die Poliklinik kommt.

Status: Das rechte Ellbogengelenk steht in stumpfwinkliger Stellung bei proniertem Vorderarm fixiert. Es ist verdickt, Konturen verwischt. Maße: 27, 28, $28 \mathrm{~cm}$ gegen 24, 25, $26 \mathrm{~cm}$ links. Die Haut ist gespannt, livide verfärbt, nur über der Streckseite zeigt sich noch eine heiße, stark gerötete Partie. Über dem Triceps ist eine zweifingerbreite, brettharte Induration. Druck aufs Olekranon besonders schmerzhaft. Bewegungen aktị unmöglich, auch im Hand- und Schultergelenk; passiven Bewegungsversuchen stellt sich ein derber, harter Widerstand entgegen; dabei lebhaftes Krachen im Gelenk. Starke Schmerzen.

Ab 26. X. Stauung. Schmerzen lassen nach. 29. X. Die harte Induration wird weicher und kleiner; passiv Spur von Bewegung. 3. XI. Letzter Rest von Entzündung fort; passiv Beugung und Streckung ca. $40^{\circ}$; aktiv Spur; Pro- und Supination ausgiebiger. Schwellung geringer 26, 26, $27 \mathrm{~cm}$. 10. XI. Streckung ca. $150^{\circ}$, Beugung $100^{\circ}$. Stauung fort. Nur noch Massage bis 20. Dez. 1907.

Entlassungsstatus: Spontan schmerzfrei; bei forcierten Bewegungen 
Zur Beh. d. Arthr. gonorrh. d, großen Gelenke mittels Stauungshyperämie. 75

noch Schmerzen. Motorische Kraft von Hand und Arm herabgesetzt. Oberarmmuskulatur atrophisch. Gelenk etwas verdickt. $26,26,27 \mathrm{~cm}$ gegen 24, $25,26 \mathrm{~cm}$. Bei Bewegungen starke rauhe Krepitation. Beugung ca. $80^{\circ}$ bis $70^{\circ}$, Streckbehinderung ca. $15^{0}-10^{\circ}$. Pronation frei, Supination etwas beschränkt.

31. H. Fr., Kaufmann, 31 J. alt. Seit 5 Jahren chronischen Tripper. Anfang Dezember starker AusfluB. 2. Dezbr. Anschwellung des linken Ellbogengelenks und Schmerzhaftigkeit bei Bewegungen. Am 12. XII. 1907 Poliklinik.

Status: Linkes Ellbogengelenk mäBig geschwollen. Maße: 24, 25, $26 \mathrm{~cm}$ gegenüber 22, 23, $24 \mathrm{~cm}$ links. Starker KapselerguB. Die Furchen zu beiden Seiten des Olekranon sind wulstartig vorgebuchtet. Die periartikulären Weichteile nicht beteiligt. Geringe entzündliche Rötung der Haut. Bewegungen aktiv und passiv nur wenig beschrankt, doch schmerzhaft. Stauung tägl. 22 Stunden. Schon nach 1 Tag Bewegungen ganz schmerzfrei.

16. XII. KapselerguB kaum noch nachweisbar. 20. XII. Nach 8 täg. Stauung geheilt entlassen. Normale Gelenkkonturen. Volle Funktion.

32. B. H., Frau, 28 J. alt. Früher gesund. Seit ca. 10 Wochen starken AusfluB. Anfang Oktober heftige Entzündung im rechten Ellbogengelenk. Behandlung mit Jodpinselungen, Umschlägen, später Schienenverbänden. Da das Gelenk mehr und mehr versteift, kommt Pat. am 7. XII. 1907 zur Poliklinik.

Status: Das rechte Ellbogengelenk ist sehr stark geschwollen. Maße: $28,30,29 \mathrm{~cm}$ gegenüber $22,22,23 \mathrm{~cm}$ links. Haut darubber nur wenig gerötet. Im Sulcus bicipitalis int. derbe, $2 \mathrm{~cm}$ breite Infiltration, die sich bis zur Mitte des Oberarms hinzieht. Das Gelenk steht in stumpfwinkliger Beugestellung fixiert. Aktive Bewegungen auch der Hand und Finger nicht ausführbar. Passiven Bewegungsversuchen setzt sich im Gelenk ein starrer, harter Widerstand entgegen; bei forcierten Bewegungen starkes Krachen.

Ab 7. XII. 190722 stündige Staung, die ein sehr feuriges Ödem hervorruft. 16. II. Schwellung zurückgegangen. Maße: 24, 26, $24 \mathrm{~cm}$. Die Rötung weicht einer lividen Verfärbung der Haut. Runzelung der Haut. Infiltration kleiner und weicher. Aktive Bewegungen im Sinne der Beugung setzen ein; Streckung nicht gebessert. 24. II. Maße: 23, 24,

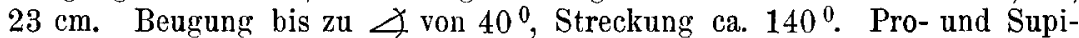
nation bis zur Hälfte. 3. I. Muskelatrophie, besonders des Biceps, tritt deutlich hervor; Maße wie links. Nur noch alle 2 Tage Stauung. Massage. 5. I. Beugung wie links. Streckung ca. $150^{\circ}$. Pro- und Supination frei. 15. I. Nur noch Streckbehinderung ca. $20^{\circ}$. Verläßt vorzeitig die Behandlung.

33. F. T., 27 J. alt. Am 6. III. 1905 Fall auf die rechte Hand und rechte Schulter. Am 7. III. Poliklinik. Diagnose: Distorsio manus et humeri dextri. 4 Wochen fixierender Verband, 2 Wochen Massage, aber dabei immer sehr starke Schmerzen. Bewegungsunfähigkeit der Schulter.

25. IV. Status: Äußerlich mäßige Schwellung. Völlige Fixation und Bewegungsunfähigkeit im rechten Schultergelenk, starke Druckempfindlichkeit im Bereich der Bicepssehne, starke andauernde Schmerzen, die in den 
Oberarm ausstrahlen, passiv jede Bewegung unmöglich. Tägl. 1 Stunde Stauung nach 8 Tagen 12 Stunden, wesentliche Besserung; Arm wird bis $25^{\circ}$ erhoben. Dabei ganz wenig Beschwerden.

Seit 12. V. Neuritis ulnaris, die trotz langer Stauung sehr schmerzluaft ist. Erst Mitte Juni Besserung; von da ab täglich 4 Stdn. Stauung bis zur vollkommnen Heilung Ende Juni. 4. VI. 1907 Nachuntersuchung. Pat. ist als Masseurin tatig. Rechtes Schultergelenk vollkommen gesund und funktionsfähig.

34. S., Kontoristin, 19 J. alt. Sonst gesund, Vor 3 Jahren angeblich Reißen in den Knien, das ohne ärztliche Hilfe fortging. Seit $3 / 4 \mathrm{~J}$. eitrigen AusfluB. Vor 14 Tagen plötzlich ohne besondere Ursache Schmerzen der linken Schulter und Bewegungsunfähigkeit. Trotz Einreibungen beständige Verschlimmerung. Am 14. III. 1905 in der Poliklinik fixierender Verband.

16. III. 1905 Status: Das linke Schultergelenk ist stark druckempfindlich, ein ErguB nicht nachweisbar; fast völlige Fixation, nur nach vorn ist eine geringe Elevation innerhalb $10^{\circ}$ möglich, sonst geht die Scapula mit, bei geringstem Bewegungsversuch sehr starke Schmerzen.

17. III. 1905 tägl. 1 Stunde Stauung. Fixierende Verbände. 20. III. Schmerzen so gering, daß Verband fortgelassen wird. Stauung gut ertragen. 30. III. Auffallend schnelle Wiederherstellung der Bewegung, dabei hier und da leichte Schmerzen. Elevation nach vorn in normaler Weise bis zur Senkrechten. Seitlich bis $70^{\circ}$ ohne Skapula mitgehen. Nur noch leichte Druckempfindlichkeit am Akromialrand. 1. IV. entlassen. 7. VII. 1907 Nachuntersuchung. Spürt noch Witterungswechsel. Schultergelenk gesund. Volle Funktion.

35. P., Frau, 37 J. alt. Früher gesund. Seit längerer Zeit Ausfluß. Seit 15. Novbr. 1907 Erkrankung der linken Schulter. Schwellung, Rötung, heftige Schmerzen und Schlaflosigkeit. Umschläge, Einreibungen ohne EinfluB. Schultergelenk wird immer unbeweglicher. Poliklinik 30. XII. 1907.

Status: Linke Schultergelenksgegend mäig geschwollen. Haut darüber unverandert; fühlt sich heiß an. Deltoideusatrophie. Der Arm hàngt schlaff am Thorax herunter. Humeruskopf ist völlig fixiert in der Pfanne; jede Bewegung, auch passiv, unmöglich. Starke Schmerzhaftigkeit.

Ab 30. XII. 22 stündige Stauung. Schmerzen lassen nach. Nachts Schlaf. 2. I. 1908. Kopf schon etwas beweglich; passiv geringe Rotation. 5. I. 1908. Aktiv Elevation in der Sagittal- und Frontalebene ca. $20^{\circ}$ ohne Scapulamitgehen. 15. I. 1908. Rasche Besserung. Arm nach vorn und seitlich ca. $50^{\circ}$. Rotation ausgiebiger. 20. I. 1908. Elevation nach vorn bis zur Senkrechten. Abduktion bis zur Horizontalen. 25. I. 1908. Kopf ganz frei beweglich. Keine Funktionsbeschränkung mehr. Massage angeraten. Entlassen. 30. I. 1908 Nachuntersuchung. Volle Funktion des linken Arms.

36. B., Frau, 42 J. alt. Früher gesund. Seit längerer Zeit Blasenkatarrh und AusfluB. Am 1. Dezbr. 1907 plötzlich Erkrankung der linken Schulter; heftige Schmerzen, Unbeweglichkeit. Behandlung mit Gipsver- 
Zur Beh. d. Arthr. gonorrh. d. großen Gelenke mittels Stauungshyperämie. 77

band 3 Wochen lang. Nach Abnahme des Verbands blieb der Arm steif. Deshalb am 30. XII. 1907 Poliklinik.

Status: Geringe Schwellung der linken Schultergelenkgegend. Haut fühlt sich hei $\beta$ an. Humeruskopf fest fixiert. Bewegungen aktiv und passiv behindert.

Ab 31. XII. Stauung, die bei der sehr empfindlichen Pat. schmerzlindernd wirkt. 8. I. 1908. Abduktion und Elevation ca. $40^{\circ}$. 16. I. 1908. Heftige Schmerzen im Verlauf des rechten Radialis und Ulnaris. Bei bloßer Berührung lautes Aufschreien. 20. I. Druckempfindlichkeit im Radialis- und Ulnarisgebiet verschwunden. 25. I. Kopf beweglicher; doch zeitweise noch Schmerzen im Gelenk. 5. II. Abduktion bis $70^{\circ}$; Elevation bis $80^{\circ}$. 10. II. AuBenrotation noch beschrankt. 15. II. Abduktion bis zur Horizontalen; Elevation bis zur Senkrechten. Rotation wie rechts. Massage. 22. II. Geheilt entlassen. Zeitweise noch Schmerzempfindung. Volle Funktion.

3\%. Schr., Verkäuferin, 21 J. alt. Früher gesund. Seit mehreren Monaten Ausfiuß. Seit Anfang Mai plötzlich erkrankt unter Fieber, Anschwellung und Rötung des linken Kniegelenks. Behandlung in einem städt. Krankenhaus mit Gipsverbänden. Nach 3 Wochen Gelenkpunktion. Am 15. VI. 1907 auf Wunsch ungebessert entlassen. Zu Hause Weiterbehandlung mit Umschlägen, Einreibungen; trotz aller Maßnahmen zunehmende Verschlimmerung, rasende Schmerzen, deshalb Poliklinik am 5. VIII. 1907.

Status: Pat. muß getragen werden. Das linke Kniegelenk ist spindelförmig aufgetrieben, Konturen verwischt. Grenzen der Patella verstrichen. Maße am oberen Rand der Patella, über die Mitte der Patella und am unteren Rand der Patella: 37, 36, $36 \mathrm{~cm}$ links, 32, 33, 32 rechts. Gelenkkapsel verdickt, die paraartikulären Weichteile teigig geschwollen, ganz das Bild eines tub. Fungus, das Gelenk fühlt sich heiß an; die leiseste Berührung schmerzhaft. Knie in geringer Beugestellung fixiert. Bewegungen aktiv und passiv ganz unmöglich.

5. VIII. Stauung. Schmerzen lassen nach, in der Nacht Schlaf. Am 6. VIII. kommt Pat. zu FuB in die Poliklinik. 14. VIII. Rasche Besserung. Spontan schmerzfrei, Schwellung geht zurück, aktiv Spur von Bewegung, passiv ziemlich ausgiebig ohne Schmerzen, sobald die Binde fortgelassen wieder Schmerzen. 15./25. VIII. Pat. bleibt aus der Behandlung fort, staut zu Hause. 25. VIII.-5. IX., poliklinisch unregelmäBig gestaut, da Pat. öfters wegbleibt. Maße: $34,35,33 \mathrm{~cm}$. Beugung bis zu $70^{\circ}$, Strecken vollständig. 5. IX. Pat. verläßt die Behandlung. 15. IX. Nachuntersuchung: In Ruhe und beim Gehen ganz schmerzfrei. Gelenkkonturen normal. Umfangsmaße wie rechts. Oberschenkelmuskulatur etwas atrophisch. Streckung vollständig, Beugung ca. $70^{\circ}-60^{\circ}$.

38. P., Frau, 27 J. alt. Stets gesund. Am 24. VI. ohne nähere Ursache Schmerzen in allen Gelenken. Das rechte FuBgelenk ist besonders schwer befallen, Behandlung mit heiBen Bädern und fixierenden Verbänden. Trotzdem Zunahme von Rötung, Schwellung und Unbeweglichkeit. Am 6. VII. 1907 Aufnahme in die Klinik.

Status: Das rechte FuBgelenk ist sehr stark geschwollen, gerötet und 
ödematös besonders über beiden Knöcheln, die Gruben zu beiden Seiten der Achillessehne verstrichen. Geringe Plantarflexion möglich, sonst alle Bewegungen behindert, lebhafte Schmerzhaftigkeit besonders bei Berührung.

3 Wochen Dauerstauung tägl. 22 Stunden bis 27. VII., sodann HeiBluft, Massage und passive Bewegungen bis zum 5. VIII. 1907. Entlassen zur Poliklinik.

Status: Pat. hinkt, rechtes FuBgelenk verdickt. MaBe oberhalb der Malleolen, über die Malleolen, über Hacken und Gelenkfurche: $28,31,23 \mathrm{~cm}$ rechts, 27, $30,20 \mathrm{~cm}$ links. Plantar- und Dorsalflexion gegenüber links ca. $10^{\circ}$ behindert; Pro- und Supination und Rotation ebenfalls etwas behindert. Leichtes Knacken im Gelenk, beim Auftreten noch Schmerzen. Vom 5. VIII.-5. IX. Massage und heiße Bäder.

5. IX. Endresultat: Rechtes FuBgelenk ist immer noch ca. $1 \mathrm{~cm}$ dicker wie links. Bewegungen im FuBgelenk so ausgiebig wie links. Kein Krepitieren mehr. Pat. schont noch den FuB beim Auftreten.

39. W. K., 27 J. alt, Kellner. Früher gesund. Seit mehreren Wochen Gonorrhoe. Überweisung des Pat. von der Augenklinik, dort wegen Conjunctivitis gonorrhoica behandelt.

14. XII. 1906 Status: Schwellung und Rötung der rechten Handgelenksgegend. Starker Gelenkserguß. Die periartikulären Gewebe kaum beteiligt. Bewegungen aktiv unmöglich, passiv sehr schmerzhaft. Arthritis serofibrinosa.

Ballonartige Schwellung des rechten Kniegelenks. Konturen verwischt. GroBer Erguß im Gelenk und in den Recessus. Tanz der Patella. Aktive Bewegung unmöglich, passiv nur unter Schmerzen. Fieberbewegungen bis 38,1.

Vom 15./29. XII. tägl. 22 Stunden Staung der Hand mit Wiederherstellung vollkommener Funktion. Währenddessen ist zum Vergleich das rechte Knie auf eine Volckmannsche Schiene gelagert. Knie am 29. XII. Status idem vom 14. XII. 1907. Ab 30. XII. Staung des Knies tägl. 22 Stunden. Am 20. I. normale Kniegelenksfunktion. Am 3. II. 1907 entlassen; Knie- and Handgelenk zeigen normale Konturen and sind vollständig funktionsfähig.

40. B., Frau, 25 J. alt. Früher nie ernstlich krank. Am 3. V. Partus. Puerperium fieberhaft. Am 11. V. hohes Fieber, Schmerzen im linken Knie- und linken FuBgelenk. Zunehmende Rötung und Schwellung beider Gelenke, Schmerzen steigern sich bis zur Schlaflosigkeit, Stauversuche ohne Erfolg; ebenso Hochlagerung mit kalten Umschlägen, deshalb Überweisung des Pat. am 3. VII. 1907 nach der chir. Klinik.

Status: Das linke FuBgelenk ist sehr stark geschwollen, die Konturen ganz verwischt, die Haut darüber sehr stark gerötet und ödematös. Die Schwellung erstreckt sich bis zur Mitte des Unterschenkels and nach vorn zu den Zehen und ist besonders stark über dem Mittelfuße. Die Strecksehnen sind stark infiltriert und auf Druck äuBerst schmerzhaft. Der FuB steht in extremster SpitzfuBstellung. Jeder Bewegungsversuch ist wegen enormer Schmerzhaftigkeit unmöglich. Das linke Kniegelenk ist ballonartig aufgetrieben, die Recessus sind prall gefüllt, die Patella tanzt. Das Knie steht fixiert in Streckstellung, Bewegungen unmöglich.

3. VII. Stauung tägl. 22 Stunden. Schon nach 24 Stunden Spur von 
Zur Beh. d. Arthr. gonorrh. d. großen Gelenke mittels Stauungshyperämie. 79

Bewegung in FuB- und Kniegelenk, Schmerzen nachgelassen. 15. VII. Das Knie kann bis zu einem $\measuredangle$ von $140^{\circ}$ gebeugt werden, die SpitzfuBstellung ist zum Teil korrigiert, Bewegungen im Fußgelenk ziemlich ausgiebig. Stauung bis 1. VIII. Vom 1./14. VIII. Heißluftbehandlung. Seit 13. VIII. geht Pat. mit 2 Stöcken im Zimmer umher. Das Kniegelenk ist bis $z u$ 1 R. beugefahig. FuBgelenk gut beweglich, noch geringe SpitzfuBstellung. 24. VIII. der Poliklinik überwiesen. Kniegelenk vollkommen beweglich. FuBgelenk noch verdickt. Maße: 18,5, 21, 26,5 cm links, 16, 19, 25,5 cm rechts, Dorsalflexion $10^{\circ}$ behindert, leichtes Krepitieren. $\mathrm{Zu}$ Hause Massieren und FuBbäder.

Nachuntersuchung am 27. Sept. 1907: Pat. hat leicht hinkenden Gang. Gelenkkonturen: Knie normal, FuBgelenk etwas verdickt. Bewegungen im Knie- und FuBgelenk vollkommen normal.

\section{Literaturverzeichnis.}

1. Bier, Weitere Mitteilungen über die Behandlung chirurgischer Tuberkulose mit Stauungshyperämie. Arch. f. klin. Chir. 1894.

2. Habs, Über Biersche Stauung. Münchner med. Wochenschr. 1903, Nr. 22.

3. Luxemburg, Über Biersche Stauung. Münchner med. Wochenschr. 1904, Nr. 10.

4. Laqueur, Zur physikalischen Behandlung der gonorrhoischen Gelenkerkrankungen. Berliner klin. Wochenschr. 1905, Nr. 23.

5. Tillmans, Über Behandlung durch venöse Stauung. Deutsche med. Wochenschr. $190 \overline{5}$, Nr. 4.

6. Tiling, The treatment of gon. Arthritis by Hyperemia. Journal of the Americ. med. Assoc. 1905, 29. April.

6. Bum, Die Behandlung der Gelenkerkrankungen mittels Stauung. Wiener med. Presse 1905, Nr. 34. 4.

8. Hirsch, Über die Behandlung der Arthritis gon. mittels Stauungshyperämie. Berliner klin. Wochenschr. 1905, Nr. 39.

9. Leyden-Lazarus, Rud. v. Leuthold-Gedenkschrift, 1. Band. Über die Behandlung der Gelenkentzündungen mit der Bierschen Stauungshyperämie.

10. Treupel, Ửber die medikamentöse und lokale Behandlung der akuten und chronischen rheumatischen und gonorrhoischen Gelenkerkrankung. Münchn. med. Wochenschr. 1907, Nr. 39.

11. Arapow, Zur Behandlung der Arthr. gon. mittels Stauungshyperämie. Referat im Zentralbl. f. Chir. 1907, Nr. 24.

12. Mathies, Zur Behandlung der Arthr. gon. Inaug.-Diss. Kiel 1907. Referat im Zentralbl. f. Chir. 1907, Nr. 39. 